\title{
Kommerzialisierung und Marktkonstitution
}

\author{
Zur politischen Konstruktion des Sportrechtemarktes*
}

Henk Erik Meier

In der gegenwärtigen Diskussion über die Kommerzialisierung der Medien wird oft die Tatsache verdrängt, dass Medienmärkte politisch konstituiert sind. Im Anschluss an Neil Fligstein konzeptionalisiert der vorliegende Beitrag die Schaffung von Marktinstitutionen als genuin politischen Prozess, in dessen Verlauf die sektoralen Akteure um eine Stabilisierung ibrer Marktinteraktionen unter Zubilfenabme staatlicher Durchsetzungspotenziale ringen. Um die Relevanz dieser theoretischen Perspektive empirisch zu verdeutlichen, rekonstruiert der Beitrag die Regulierung des Sportrechtemarktes. Dabei wird nachgewiesen, dass die sektoralen Akteure versucht haben, den Sportrechtemarkt unter Mobilisierung politischer Macht oder rechtlicher Ressourcen in ibrem Interesse zu strukturieren. Die Komplexität und Interdependenz der Regulierungsprobleme fübrte dazu, dass diese in drei segmentierten Politikarenen bearbeitet wurden, die durch differierende kognitive Referenzrabmen charakterisiert und in unterschiedlichem Maße offen für die sektoralen Interessen waren. Im Ergebnis dieser politischen Prozesse weist der Sportrechtemarkt ein sehr hohes Regulierungsniveau auf, das sich zu Lasten der Einnabmeinteressen der Sportrechteinhaber auswirkt. Die Konkurrenz unterschiedlicher Regulierungsansätze hat dazu geführt, dass der Sportrechtemarkt als noch nicht hinreichend stabilisiert angeseben werden kann.

Keywords: Medienpolitik, Kommerzialisierung, Marktkonstituierung, Marktregulierung, Sportrechtemarkt, Zentralvermarktung, Schutzlistenregelung

\section{Einleitung}

Die Kommerzialisierung der Medien wird häufig als eindimensionaler Prozess der Durchsetzung ungehemmter ökonomischer Rationalitäten konzeptionalisiert. Dabei wird nicht hinreichend gewürdigt, dass es sich auch bei liberalisierten Medienmärkten um politisch konstituierte Industrien handelt, die auf nichtökonomischen Koordinationsmechanismen basieren (Lütz/Czada 2000).

Während dieser Aspekt in den vorwiegend systemtheoretisch inspirierten Überlegungen zur Kommerzialisierung der Medien eine geringe Rolle spielt (Meier/Jarren 2001), hat der amerikanische Wirtschaftssoziologe Neil Fligstein (2001) einen politischkulturellen Ansatz für die Analyse von Marktinstitutionen formuliert. Dieser Ansatz nimmt die zentrale Einsicht der ökonomischen Soziologie auf, dass wirtschaftliches Handeln als eingebettet in umfassendere soziale Strukturen verstanden werden muss. Dabei haben einerseits die sozialen Beziehungen zwischen Marktakteuren eine erhebliche Bedeutung für die Funktionsweise und die Ergebnisse von Märkten (Granovetter

* Der vorliegende Beitrag stellt Ergebnisse des Forschungsprojektes „Die politische Regulierung professioneller Sportligen“ vor, das vom Bundesinstitut für Sportwissenschaft finanziell gefördert wurde (VF 0407/09/40/2003-2004). Der Autor ist Mitarbeiter der Kommission zur Ermittlung des Finanzbedarfs der Rundfunkanstalten (KEF), der vorliegende Artikel gibt nur seine persönliche Meinung wieder. 
1985). Andererseits besteht nach Ansicht der ökonomischen Soziologie eine Interdependenz zwischen Marktinstitutionen sowie kulturellen, politischen und sozialen „background institutions“ (DiMaggio 1994). Fligstein (2001) entwickelt vor allem die institutionenökonomischen Überlegungen North’ (1990) weiter, nach denen eigennutzorientierte sektorale und staatliche Akteure durch ihre Interaktionen Regeln für Markttransaktionen schaffen, die - intendiert oder nicht - die Marktentwicklung langfristig prägen, ohne deshalb notwendig allokativ oder produktiv effizient zu sein. Fligstein (2001) konzeptionalisiert die Konstituierung von Marktinstitutionen dabei als genuin politischen Prozess, in dessen Verlauf die sektoralen Akteure nicht um effiziente Marktarrangements, sondern um eine günstige Stabilisierung ihrer Marktinteraktionen unter Zuhilfenahme staatlicher Durchsetzungspotenziale ringen. Für die Durchsetzungsfähigkeit konkurrierender sektoraler Interessen spielen dabei nicht nur ihre unterschiedliche Konfliktfähigkeit, sondern auch die Policy-Präferenzen der politischen Akteure, die Spielregeln der segmentierten politischen Entscheidungsarenen sowie historische Pfadabhängigkeiten und Kontingenzen eine erhebliche Rolle (Thelen 1999). Die ökonomische Soziologie nimmt damit eine interaktionsorientierte Perspektive ein und versucht, die Entstehung von Marktinstitutionen als das Produkt der Interaktion zwischen sektoralen und politischen Akteuren zu rekonstruieren, von denen jeder über je eigene Problemdeutungsmuster, individuelle und institutionelle Eigeninteressen sowie normative Präferenzen und Handlungsressourcen verfügt (Fligstein 2001).

Der vorliegende Beitrag versucht, das Erklärungspotenzial dieses Ansatzes am Beispiel der politischen Konstruktion des Sportrechtemarktes nach der Deregulierung der Fernsehmärkte zu demonstrieren. Ziel ist es zu zeigen, dass sich die Konturen des Sportrechtemarktes als Ergebnis der eigennutzorientierten Interaktionen interessierter Akteure rekonstruieren lassen, in deren Folge einerseits ökonomische Rationalitäten teilweise außer Kraft gesetzt werden und andererseits ein teilweise inkonsistentes Regulierungsregime entstanden ist. Entsprechend der interaktionsorientierten Perspektive des Ansatzes werden bei der folgenden Darstellung Akteursinteressen und -strategien, Entscheidungsprobleme und -arenen sowie Entwicklungspfade und Interaktionsergebnisse im Vordergrund stehen. Ziel ist es, auf der Basis einer detaillierten deskriptiven Rekonstruktion „analytic narratives“, also konzeptionell strukturierte „Erzählungen“ über kohärente Sequenzen motivierter Handlungen, zu formulieren (Aminzade 1992; Bates et al. 1998). Das Datenmaterial der vorliegenden Untersuchung besteht aus amtlichen Dokumenten, der einschlägigen Sekundärliteratur, Stellungnahmen und Publikationen der beteiligten Akteure sowie der Berichterstattung in Tageszeitungen und Nachrichtendiensten. Darüber hinaus wurden über dreißig Interviews mit Experten aus dem Untersuchungsfeld sowohl auf nationaler als auch auf europäischer Ebene geführt.

Die hier vorgenommene Beschränkung auf den Sportrechtemarkt bietet sich deshalb an, weil es sich hierbei um einen der wichtigsten Beschaffungsmärkte der audiovisuellen Medienindustrie handelt, dessen Kontrolle im vitalen Interesse der Medienunternehmen liegt. Die wirtschaftliche und strategische Bedeutung von Sportübertragungsrechten wird an dem sprunghaften Anstieg der Rechtspreise nach der Deregulierung der Fernsehmärkte deutlich, der bis zum Platzen der Digitalblase anhielt (Abb. 1).

Dass die politische Konstruktion des Sportrechtemarktes vor allem durch Auseinandersetzungen um die Vermarktung von Fußballübertragungsrechten angestoßen wurde, resultiert daraus, dass Fußball auf dem europäischen Fernsehmarkt ein wichtiger Träger sozialer Identitäten (Giulianotti 1999) und gleichzeitig die mit Abstand ökonomisch erfolgreichste Sportart ist (Barros et al. 2002). Dabei hatte die besondere Architektur des europäischen Sportmodells zur Folge, dass die Deregulierung der Fernsehmärkte er- 
Abbildung 1: Preisentwicklung ausgewäblter Sportrechte ${ }^{1}$

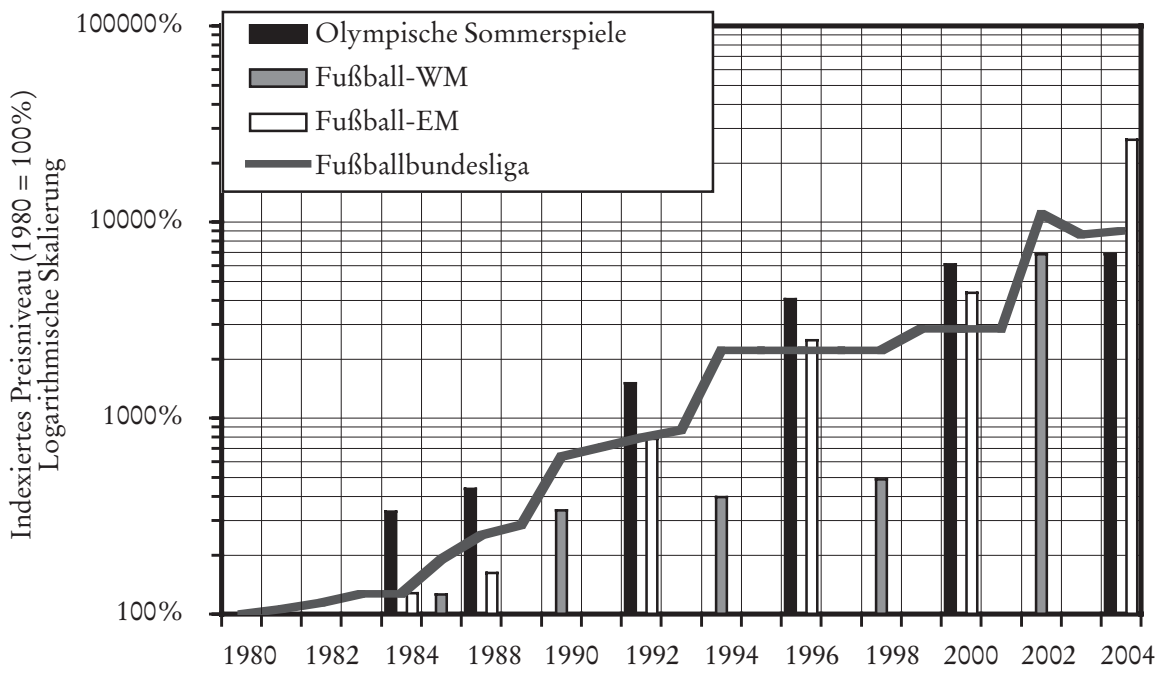

Quelle: Amsinck 1997; Chung 2001; Elter 2002.

hebliche Rückwirkungen auf die „Branchenstruktur“ des europäischen Profifußballs hatte, da sie den Optionenspielraum und das Einflusspotenzial einzelner Akteure im komplexen Abhängigkeitsgefüge innerhalb des Sports veränderte.

Während Sportrechte per se keine kommerziellen Güter sind, die ohne rechtliche Strukturierung vermarktet werden können, zeichnete sich der Sportrechtemarkt bis zur Deregulierung des Fernsehmarktes durch ein relativ geringes Regulierungsniveau aus. Das deutsche Recht kennt im Unterschied zur amerikanischen Rechtslage bspw. kein ausdrücklich normiertes urheberrechtliches Schutzrecht für die Veranstalter von Sportveranstaltungen (Elter 2002). Das Fernsehübertragungsrecht stellt nur ein schuldrechtliches Verwertungsrecht dar, über das jedoch ähnlich wie über ein Leistungsschutzrecht ganz oder teilweise verfügt werden kann (Petersen 2001). Die dogmatische Herleitung dieses Rechts aus dem Hausrecht oder dem Wettbewerbsrecht war umstritten und hatte weit reichende Implikationen für die Rolle, die den Sportverbänden in der Wertschöpfungskette und damit bei der Vermarktung der Wettbewerbe zugewiesen wurde (Hannamann 2001).

Das geringe Regulierungsniveau auf dem Sportrechtemarkt musste zwangsläufig zur Folge haben, dass der Liberalisierung der Fernsehmärkte der Aufbau neuer regulativer Strukturen folgte. Für die Kennzeichnung solcher Prozesse auf liberalisierten Märkten hat sich in den Sozialwissenschaften im Anschluss an Majone (1996) der Terminus „ReRegulierung" durchgesetzt. Dieser Begriff kennzeichnet allerdings den gesamten Prozess der Schaffung neuer regulativer Strukturen in einstigen wirtschaftlichen Ausnah-

1 Für die Fußball-Weltmeisterschaften stellt 1982 das Basisjahr der Indexierung dar. Der Preisindex für die Fußball-EM bezieht sich nur auf die wirtschaftlich bedeutendsten europäischen Rechte. 
mebereichen, d. h. sowohl die marktschaffende ordnungspolitische Rahmengesetzgebung als auch die marktkorrigierende einzelfallbezogene Kontrolle des Marktgeschehens durch (verselbstständigte) Aufsichtsbehörden. Er bleibt somit gegenüber juristischen Definitionen, aber auch dem engen Regulierungsbegriff der amerikanischen Forschung unscharf (Noll 1985). Aus diesem Grund, aber auch wegen der technokratischen Konnotationen des Re-Regulierungsbegriffes, wird hier von der politischen Konstitution des Sportrechtemarktes gesprochen. Für diesen Prozess war entscheidend, dass die spezifischen Regulierungsprobleme in die Schnittmenge dreier segmentierter Politikarenen fielen, die nicht nur durch unterschiedliche Interessenkonstellationen, sondern auch durch stark differierende Policy-Paradigmen gekennzeichnet sind.

Der vorliegende Beitrag skizziert zunächst die grundlegende sektorale Interessenkonstellation auf dem Sportrechtemarkt (2.) und diskutiert dann die relevanten Präferenzen der politischen Akteure für die Strukturen des Sportrechtemarktes (3.). Anschließend werden ausgewählte Entscheidungsprozesse zur Konstruktion des Sportrechtemarktes dargestellt (4.), bevor den Implikationen des vorliegenden Beitrages für die Debatte über die Kommerzialisierung des Mediensystems nachgegangen wird (5.).

\section{Die sektorale Interessenkonstellation}

Die strategische Bedeutung von Sportrechten auf dem audiovisuellen Medienmarkt resultiert nicht nur aus der Reichweitenstärke und damit der kommerziellen Attraktivität von Sportübertragungen. Vielmehr stellen Sportereignisse für Fernsehanbieter wenig elastische und schwer imitierbare Inputfaktoren dar. Die Senderechte an Sportveranstaltungen zeichnen sich durch einen starken Aktualitätsbezug aus, da sie aufgrund der Relevanz der Unsicherheit über den Ausgang des sportlichen Wettbewerbs für den Konsum faktisch nicht lagerfähige Güter sind. Darüber hinaus weisen sie eine geringe Substitutionselastizität auf. Schließlich unterliegen Sportsendungen wie alle Programmangebote des Fernsehens einer erheblichen Fixkostendegression (Enderle 2000; Gaustad 2000; Kruse 2000; Solberg 2002).

In ihrer Kombination konstituieren diese Besonderheiten von Sportrechten für die Fernsehanbieter starke Anreize, möglichst umfangreiche und exklusive Übertragungsrechte zu erwerben. ${ }^{2}$ Die exklusive Verwertung von Sportübertragungsrechten verspricht die größten Einnahmen und erlaubt es den Medienanbietern erst, jene Imagegewinne zu erreichen, die insbesondere für den Marktzutritt notwendig sind (Kruse 1991). Als Beispiel für einen erfolgreichen Marktzutritt über den Erwerb attraktiver Sportrechte galt vor allem die im Besitz von Rupert Murdoch befindliche News Corporation, die attraktive Übertragungsrechte sowohl in den USA als auch in Großbritannien als „battering ram" genutzt hatte, um sich auf diesen Märkten zu etablieren (Spink/Morris 2000).

Allerdings verstärkt der Exklusiverwerb nicht-imitierbarer Sportrechte die Konzentrationstendenzen auf dem Fernsehmarkt. Der Erwerb exklusiver Sportrechte schien das Fernsehen mit Beginn der Digitalisierung sogar in einen „winner takes all“-Markt zu transformieren, auf dem ein Unternehmen eine dominante Position erringen konnte, während seine Konkurrenten um das wirtschaftliche Überleben fürchten mussten (Sol-

2 Der Erwerb von Paketübertragungsrechten an Ligawettbewerben oder Sportgroßereignissen hat für die Fernsehanbieter schließlich auch den Vorteil, dass sie sich auf diese Weise gegen die Risiken des sportlichen Erfolges einzelner Akteure oder Teams versichern können (Enderle 2000). 
berg 2002). Angesichts solcher Marginalisierungsszenarien besteht für die Fernsehanbieter ein erhebliches Interesse an der Kontrolle der Vermarktungspraktiken der Sportrechteinhaber.

Die Deregulierung der Fernsehmärkte entsprach zwar zunächst den Einnahmeinteressen der Sportverbände, die zum Teil erhebliche Erlössteigerungen bei der Verwertung ihrer Übertragungsrechte erzielen konnten. Diese „Umfeldkommerzialisierung“ hatte jedoch erhebliche Auswirkungen auf die Interessenkonstellationen innerhalb des organisierten Sports. Das europäische Sportmodell zeichnet sich im Gegensatz zu den USProfiligen, die rein kommerziell orientierte Profitgenossenschaften darstellen, durch die Anbindung des Profibereichs an den Amateursport aus. Diese Anbindung wird u. a. dadurch gewährleistet, dass die Sportverbände ein Monopol auf die Veranstaltung von Sportwettkämpfen haben (Hannamann 2001). Dies ermöglicht den Sportverbänden den Zugriff auf die Vermarktungspolitik und die Einnahmen des Profisports, aber auch die Regulierung seiner Ausbildungs- und Rekrutierungspolitik. Darüber hinaus werden die Profiligen auch über ein Relegationssystem an den Amateursport angebunden (EUKommission 1998a). Dieses institutionelle Arrangement ist, wie das US-amerikanische Beispiel zeigt, keineswegs selbstverständlich, da der Profisport für seinen kommerziellen Erfolg weder zwingend einen Verband noch einen Amateurbereich benötigt (Franck 1995). Die Umfeldkommerzialisierung des Sports ließ daher trotz enormer Einnahmesteigerungen die Verteilungskonflikte zwischen Sportverbänden und Profiligen eskalieren. Diese Verteilungskonflikte resultieren daraus, dass sowohl die Sportverbände als auch die Proficlubs als Einnahmemaximierer agieren. Während die Sportvereine auf diese Weise versuchen, die für ihre jeweilige Sportart verfügbaren Mittel zu erhöhen (exemplarisch: UEFA 1998), geht das einnahmemaximierende Verhalten der Proficlubs darauf zurück, dass sportlicher Erfolg in Mannschaftssportarten trotz der erheblichen Relevanz von Zufallsfaktoren grundsätzlich über die Zusammenstellung von Spieltalent käuflich ist (Franck 1995). Die Proficlubs drängten daher auf einen höheren Anteil an den gestiegenen Einnahmen. Darüber hinaus kam es zwischen Proficlubs und Sportverbänden zu Konflikten über die Vermarktungspolitik der Profiligen. Die Fußballverbände hatten ihr Vermarktungsmonopol stets dazu genutzt, das Angebot an Live-Fußball zu verknappen, weil sie von einer Substitutionskonkurrenz zwischen Fernsehübertragungen und Stadionbesuch ausgingen und eine Gefährdung der aktiven Ausübung des Fußballsports durch ein zu großes Angebot an Fußballübertragungen vermeiden wollten (Quitzau 2003). Weitere Auffassungsdifferenzen über die Vermarktungspolitik resultierten daraus, dass Sportverbände und Profiligen vor einem schwierigen "trade-off“ zwischen der Maximierung der Sichtbarkeit ihres Sports und der (kurzfristigen) Fernseheinnahmen stehen, weil der Wert von Sportübertragungsrechten wesentlich von „sozialen Netzwerkexternalitäten“ abhängt (Solberg 2002). Top-Sportereignisse gelten als ein Beispiel für interdependente Präferenzen, bei denen ein Konsument Nutzen durch die Konsumption eines Produktes durch andere erhält. Eine Angebotsverknappung oder eine Verlagerung in das Pay-TV kann daher den „Konversationswert“ und damit seine langfristigen Einnahmepotenziale reduzieren (Boardman/Hargreaves-Heap 1999). Zwischen den eher sportpolitischen Zielsetzungen verpflichteten Sportverbänden und den einnahmemaximierenden Proficlubs kam es daher auch zu Differenzen über die Gewichtung der Ziele der langfristigen medialen Präsenz und der kurzfristigen Einnahmemaximierung. Dieses Bündel von Interessenkonflikten führte dazu, dass die Anbindung des Profibereichs an den Amateursport nach der Deregulierung der Fernsehmärkte zunehmend in Frage gestellt wurde.

Das Interesse der Proficlubs an einer individuellen Einnahmemaximierung hat auch 
für die Profiligen die Verschärfung der internen Verteilungskonflikte zwischen ertragsstarken und -schwachen Clubs zur Folge. Die Umfeldkommerzialisierung des Sports löst in Mannschaftssportarten einen sich selbst verstärkenden Prozess der individuellen Einnahmemaximierung durch die einzelnen Clubs aus, die ihren wirtschaftlichen Erfolg maximieren müssen, um sportlich wettbewerbsfähig zu bleiben. Dabei gehen Sportökonomen und Sportverbände gleichermaßen davon aus, dass die sportliche Ausgeglichenheit einer Liga die Voraussetzung für ihren langfristigen kommerziellen Erfolg ist. Diese These basiert auf der Annahme, dass die Attraktivität des Meisterschaftsrennens für den Zuschauer vermeintlich bei einer hohen Unsicherheit über den Ausgang maximiert wird (Franck 1995; Schellhaaß/Enderle 1999). Dieses Unsicherheitsargument stellt die zentrale Rechtfertigung für die Schaffung von Umverteilungsmechanismen innerhalb der Profiligen dar und bildet den Hintergrund für die Forderung nach ihrer wettbewerbsrechtlichen Sonderbehandlung. Die Bildung von Kollektivvermarktungssystemen wird als Ausfluss der spezifisch assoziativen Konkurrenz in professionellen Ligen verstanden, die ligainterne Umverteilungsmechanismen erfordert (Scully 1995). Allerdings ist die Erforderlichkeit ligainterner Umverteilungsmechanismen und Kollektivvermarktungssysteme kontrovers diskutiert worden. Zunächst erweist sich die Unsicherheitshypothese als empirisch umstritten (Überblicke über die internationale empirische Forschung bei: Cairns et al. 1986; Downward/Dawson 2000; Szymanski/Kuypers 1999; Dobson/Goddard 2001). Darüber hinaus wird seit El Hodiri und Quirk (1971) debattiert, ob Umverteilungsarrangements überhaupt Sinn machen, wenn sich eine Liga aus Teams mit (stark) unterschiedlichem Ertragspotenzial zusammensetzt. Tatsächlich nähren die einschlägigen empirischen Untersuchungen zu den Umverteilungsarrangements der US-Profiligen Zweifel an deren Effektivität bei der Herstellung eines ausgeglichenen sportlichen Wettbewerbs (Downward/Dawson 2000; Fort/Quirk 1995; Vrooman 1995). So haben prominente amerikanische Sportökonomen vor allem hervorgehoben, dass von den Kollektivvermarktungssystemen Mengenverknappungseffekte ausgehen, die es den Ligen erlauben, beeindruckende Monopolpreise zu erzielen (Noll 1982; Quirk/Fort 1999).

Diese Kontroverse kann und soll im vorliegenden Beitrag nicht entschieden werden. Für ein Verständnis der Regulierungsprozesse genügt es festzustellen, dass sich im Bereich der Profiligen außerordentlich komplexe wettbewerbspolitische Fragen stellen, die kontrovers beurteilt werden. Dies wird nicht zuletzt an der Entwicklung der amerikanischen Rechtsprechung zu Kartellbildungen im Profisport deutlich, die sich unter dem Eindruck der Chicagoer Schule zunehmend von einer restriktiven Betrachtung horizontaler Kooperationsarrangements in den Ligen gelöst hat (Weiler/Roberts 1998, Kap. 3; Wong 2002, Kap. 14).

Für ein Verständnis der Entscheidungsprozesse ist relevant, dass der europäische und der deutsche Fußballverband die Verteilungskonflikte unter den Proficlubs vor der Deregulierung der europäischen Fernsehmärkte dadurch latent hielten, dass sie unter Berufung auf ihr traditionelles Veranstaltermonopol ein Zentralvermarktungsregime praktizierten, das eine solidarische Umverteilung der Einnahmen unter den Profivereinen vorsah (UEFA 1998). Freilich wurden die Konflikte über diese Einnahmeteilung nicht zuletzt durch die von der UEFA betriebene Kommerzialisierung der europäischen Clubwettbewerbe gesteigert. Das Einnahmepotenzial dieser Wettbewerbe und ihr sportliches Prestige erhöhten die Anreize für die Spitzenvereine, Umverteilungsarrangements in den nationalen Ligen zu ihren Gunsten zu verändern, um die Risiken der sportlichen Qualifikation zu minimieren und die europäische Wettbewerbsfähigkeit ihres Spielkaders zu sichern (Brown 2000). 


\section{Das Spektrum der relevanten politischen Präferenzen}

Diese komplexe sektorale Konfliktkonstellation traf auf ein Bündel mehrdimensionaler und teilweise konkurrierender politischer Präferenzen. Die Vermarktungspraktiken der Sportverbände widersprachen wettbewerbspolitischen Zielsetzungen, weil die Verbände nicht nur eine klassische Angebotsverknappung betrieben, sondern ihr Angebotsmonopol auch dazu nutzten, um nach der Deregulierung des Fernsehmarktes Monopolpreise zu erzielen und sportliche Risiken auf die Rechteverwerter abzuwälzen. Die von den Sportverbänden betriebene Zentralvermarktung ermöglichte erst einen umfangreichen Exklusiverwerb und begünstigte damit die Konzentrationstendenzen im Medienmarkt (Stopper 1997). Dabei stellten sich einige Wettbewerbsbehörden, unter ihnen das deutsche Bundeskartellamt (BKartA) und die europäische Wettbewerbsdirektion, auf einen „orthodoxen“ wettbewerbspolitischen Standpunkt und interpretierten die Zentralvermarktungsregime als unzulässige Angebotskartelle. Sie identifizierten die Proficlubs als die eigentlichen Veranstalter - und damit: Rechteinhaber - im Profisport (Parlasca 1993).

Diese wettbewerbsrechtliche Deutung der Vermarktungspraktiken der Sportverbände widersprach freilich diametral dem Paradigma der Sportpolitik, die die Kartellbildungen im Sport für unentbehrlich hielt (Hannamann 2001). Allerdings war die Kommerzialisierung des Profisports aufgrund ihrer Rückwirkungen auf das europäische Sportmodell auch für die Sportpolitik nicht unproblematisch. Zwischen den Sportverbänden und den nationalen Sportpolitikern herrscht breiter Konsens über die Vorzüge des europäischen Sportmodells (Heinemann 1996). Die Sportpolitiker gehen davon aus, dass ihre Funktionserwartungen an den Sport, die neben gesundheitspräventiven vor allem sozialintegrative Funktionen umfassen, am besten durch eine enge Verbindung zwischen Profi- und Amateursport und die breite Sichtbarkeit sportlicher Spitzenleistungen erfüllt werden (EU-Kommission 1998a; Bundesregierung 2002). Für die Bewertung wettbewerbsrechtlicher Interventionen ist darüber hinaus relevant, dass sowohl Sportverbände als auch Sportpolitik das Paradigma der Autonomie des Sports vertreten, wonach staatliche Interventionen in den Sportbereich als dysfunktional anzusehen sind, weil sie die Fähigkeit der Sportorganisationen zur Aktivierung ehrenamtlichen Engagements gefährden könnten (Heinemann 1996).

Die Kommerzialisierung des Sports warf jedoch die Frage auf, ob der Sport seine Breitenwirksamkeit beibehalten würde, da die Einnahmemaximierungsstrategien der Sportverbände und Proficlubs wesentlich darauf abzielten, die inelastische Nachfrage der Fans auszubeuten. Für die sportpolitische Bewertung der Kommerzialisierung sind dabei die vermuteten sozialintegrativen Effekte gemeinsam geteilter Fernseherlebnisse relevant. Die sozialen Netzwerkexternalitäten des Fernsehkonsums von Sportübertragungen schaffen in den Augen der politischen Anspruchsgruppen des Sports jene seltener werdenden Gemeinschaftserlebnisse, die als unentbehrlich für die soziale Kohäsion der Gesellschaft gelten. Die Entwicklung vom frei empfangbaren terrestrischen Fernsehen zu einem fragmentierten Konsum von Fernsehsport über Pay-TV-Angebote markiert aus dieser Perspektive eine wichtige Zäsur für die mediale Konstruktion der Nation als vorgestellter Gemeinschaft (Williams 1994; Blain et al. 1993).

Über diese sozialintegrative Aufwertung des passiven Sportkonsums stellt sich die Frage, ob die Kommerzialisierung des Fußballs nicht zur Exklusion jener traditionellen Fans führt, die sich weder die gestiegenen Stadionpreise noch ein Pay-TV-Abonnement leisten können (Conn 1999). Adressat dieser Befürchtungen war in Deutschland vorrangig die Medienpolitik. Für die medienpolitische Reaktion auf die Kommerzialisie- 
rung des Sportrechtemarktes ist einerseits prägend, dass - wie auch Regulierungsinitiativen in den USA zeigen - für demokratische Politiker erhebliche Anreize bestehen, sich gegenüber den Zugangsinteressen eines breiten Teils der Wählerschaft responsiv zu zeigen (Cox 1995).

Andererseits konkurrieren diese Zugangsinteressen mit ordnungs- und wirtschaftspolitischen Zielen. So war der Erwerb attraktiver Sportrechte für die erfolgreiche Etablierung des von den liberal-konservativen Kräften betriebenen deutschen Privatfernsehens notwendig. Prinzipiell entsprach die Kommerzialisierung des Sportrechtemarktes auch der ordnungspolitischen Vision der Durchsetzung von Marktmechanismen im audiovisuellen Sektor. Zudem sollte der enorme Investitionsaufwand für die Etablierung neuer Medientechnologien die Frage aufwerfen, ob die Zugangsinteressen der Fans nicht zugunsten einer besseren Refinanzierbarkeit der neuen Technologien in den Hintergrund treten sollten (Szymanski 2000). Die drohende Marginalisierung öffentlicher Anbieter auf einem kommerzialisierten Sportrechtemarkt widersprach dagegen einer „Public Service“-Vision des Rundfunks.

\section{Analyse ausgewählter Entscheidungsprozesse zur Konstruktion des Sportrech- temarktes}

In der folgenden Analyse ausgewählter Entscheidungsprozesse zur Konstruktion des Sportrechtemarktes werden zunächst Entscheidungsanlässe und -arenen skizziert, um dem Einfluss struktureller Faktoren auf die Interaktionsergebnisse nachzugehen. Die chronologische Darstellung der Interaktionsverläufe soll verdeutlichen, dass neben den strukturellen Faktoren auch Gelegenheitsfenster sowie die Sequenzierung und die Interaktionseffekte zwischen verschiedenen Prozessen die Interaktionsergebnisse prägten (dazu: Thelen 1999).

\subsection{Die Durchsetzung eines nationalen Kurzberichterstattungsrechts im medienpoliti- schen Verbandlungssystem der Länder}

\subsubsection{Entscheidungsanlässe und-arenen}

Die Auseinandersetzung um die Verfügungsrechte der Inhaber attraktiver Sportrechte auf einem liberalisierten Fernsehmarkt begannen bereits vor dem Marktzutritt privater Anbieter, als sich Mitte der 70er Jahre die Teil-Deregulierung des Rundfunks abzeichnete. Die öffentlich-rechtlichen Anstalten befürchteten, dass ihre sinkende Nachfragemacht und das höhere Finanzierungspotenzial privater Wettbewerber ihnen dauerhaft den Zugang zu attraktiven Programminhalten abschneiden und sie damit marginalisieren würde (Meier 2003). Sie versuchten daher zunächst, eine Interpretation der verfassungsrechtlichen Rundfunkfreiheit durchzusetzen, wonach die Anstalten auf die bis dahin durch die Sportverbände freiwillig gewährten Kurzberichterstattungsrechte auch ohne Vorhandensein einer gesetzlichen Normierung einen verfassungsunmittelbaren Rechtsanspruch hatten. Dabei beriefen sie sich auf die Informationsbeschaffungsprivilegien der Massenmedien (Fuhr 1976; Kübler 1978). Das bedeutete nichts weniger, als dass die Verfügungsgewalt von Sportrechteinhabern aufgrund der besonderen öffentlichen Bedeutung ihres „Produktes“ beschränkt werden sollte. Sportübertragungen sollten partiell zu einem quasi-öffentlichen Gut gemacht werden, für das das preisliche Ausschlussprinzip nicht gelten sollte (Kruse 1991). Die ARD bestritt zudem die Existenz von Hörfunkrechten mit dem Argument, dass die Hörfunkberichterstattung genau wie 
die Presseberichterstattung nahezu ausschließlich aus der Eigenleistung des berichtenden Reporters bestehe (Kübler 1978). Die Argumentation der Anstalten, dass ihnen in Anknüpfung an presserechtliche Regelungen ein originäres Zutrittsrecht für öffentliche Veranstaltungen und eine unentgeltliche Kurzberichterstattung in Bewegtbildern verfassungsunmittelbar zustünde, wurde jedoch in der juristischen Diskussion als inakzeptable Maximalforderung angesehen, die zu tief in die Verwertungsrechte der Veranstalter eingriff (Tettinger 1986; Badura 1989; Lauktien 1992).

Die Anstalten verstärkten daher ihre politischen Bemühungen um die einfachgesetzliche Verankerung eines Kurzberichterstattungsrechts. Sie versuchten, ihr Anliegen im medienpolitischen Verhandlungssystem der Länder durchzusetzen. Dies war insoweit rational, als dass innerhalb dieser spezialisierten Politikarena die wirtschaftlichen Interessen der Sportrechteinhaber von geringerem politischen Gewicht waren, weil die Regulierung der Medienanbieter und des Medienangebots im Vordergrund stehen. Gleichzeitig konnten die Anstalten auf die Unterstützung der sozialdemokratischen Medienpolitik hoffen, zu deren zentralen Zielen in den Verhandlungen über eine duale Rundfunkordnung die Aufrechterhaltung eines starken öffentlich-rechtlichen Rundfunks zählte. Die SPD fürchtete, durch private Fernsehanbieter in der Berichterstattung benachteiligt zu werden (Gellner 1990; Tonnemacher 1996). Allerdings waren die Hürden für die Durchsetzung des Anliegens der Anstalten im medienpolitischen Verhandlungssystem zugleich sehr hoch, da die Verabschiedung bundeseinheitlicher Regelungen den Konsens aller Landesregierungen erfordert. Der ordnungspolitische Grundsatzkonflikt über die Zulassung privater Anbieter, die von den unionsgeführten Ländern vorangetrieben wurde, führte jedoch zu einer erheblichen medienpolitischen Polarisierung und $\mathrm{zu}$ außerordentlich langwierigen Verhandlungen über die duale Rundfunkordnung (Gellner 1990). Diese Entwicklung minderte zugleich die Durchsetzungschancen des Vorhabens von ARD und ZDF.

Allerdings sind die Policy-Präferenzen der Länder als zentrale medienpolitische Akteure nicht nur durch unterschiedliche ordnungs- und standortpolitische Kalküle geprägt (dazu: Tonnemacher 1996). Vielmehr verfolgen die Länder die gemeinsame „MetaPräferenz", keine rundfunkpolitischen Kompetenzen an den Bund abzutreten. Sie kooperieren daher häufig, wenn der Bund Versuche unternimmt, in ihren Kompetenzbereich vorzustoßen (Scharpf 1994). Diese „Meta-Präferenz“ kam in der Entscheidung über die Kurzberichterstattung deshalb zum Tragen, weil die Sportverbände das Bundesinnenministerium als „ihr“ Sportministerium einschalteten, um sich gegen das Anliegen der Rundfunkanstalten zu wehren. Für die Sportverbände lag die Einschaltung des BMI zum einen aufgrund der stark klientelistischen Strukturen der sportpolitischen Entscheidungsarena nahe. Zum anderen einte beide Seiten das Interesse an einer Steigerung der Einnahmen des Sports.

\subsubsection{Interaktionsverläufe und-ergebnisse}

Zunächst scheiterte das Vorhaben einer gesetzlichen Verankerung des Kurzberichterstattungsrechts im ersten Rundfunkstaatsvertrag von 1987 am Widerstand der unionsgeführten Länder, die von der Unentbehrlichkeit attraktiver Übertragungsrechte für einen erfolgreichen Marktzutritt der Privatanbieter ausgingen (Seither 1993). Die politischen Rahmenbedingungen änderten sich jedoch entscheidend 1988, als die über den Bertelsmann-Konzern mit dem Privatsender RTLplus verbundene Ufa dem DFB ein Angebot zum Erwerb der Übertragungsrechte an der Fußball-Bundesliga unterbreitete. Da die ARD das Ende ihrer traditionsreichen „Sportschau“ befürchtete, versuchte 
sie, Druck auf den DFB auszuüben, von diesem Vertrag abzusehen. Dabei spielte das Argument der damals noch geringen technischen Reichweiten der privaten Anbieter eine zentrale Rolle. Die ARD warnte den DFB vor dem Ausschluss einer Zuschauermehrheit vom Fußball und drohte für den Fall einer Nicht-Berücksichtigung mit dem exzessiven Gebrauch des vermeintlich verfassungsrechtlich gebotenen Rechts auf unentgeltliche Kurzberichte (epd 23.04.88). Der drohende Zuschauerausschluss rief auch Politiker aller Parteien auf den Plan (vgl. epd 21.05.88). Den Anstalten gelang es nun, ihre Beschaffungsinteressen mit den Zugangsinteressen einer Bevölkerungsmehrheit zu verknüpfen und die Länder zu konkreten Initiativen zur Verankerung des Kurzberichterstattungsrechts zu bewegen, das allen Fernsehanbietern zustehen sollte. Den Anstalten kam entgegen, dass die bayerische Landesregierung von ihrer ablehnenden Haltung Abstand nahm, als klar war, dass die in Bayern ansässigen privaten Veranstalter nicht in einen Vertrag mit dem DFB eintreten würden. Bei ihren Regelungsbemühungen behielten die Rundfunkreferenten die Orientierung an presserechtlichen Zugangsprivilegien bei, um die primär medienpolitische Stoßrichtung ihres Vorhabens zu unterstreichen (Hartstein/Kuch 1988).

Die DFB-Führung fühlte sich durchaus dem Anliegen der breiten Empfangbarkeit verpflichtet und erwies sich gegenüber dem öffentlichen und politischen Druck als responsiv. Innerhalb des DFB drängten die Vertreter der Bundesliga aber auf den weitaus höher dotierten Vertragsabschluss mit der Ufa (vgl. dazu: epd 27.04.88; epd 18.05.88). Im Ergebnis entschied sich die Verbandsführung im Mai 1988 für eine komplexe Vertragslösung, die zwar eine Rechtevergabe an die Ufa vorsah, gleichzeitig aber auch auf die Schaffung einer klaren exklusiven Verwertungshierarchie zugunsten von RTLplus verzichtete und $A R D$ und $Z D F$ die vorläufige Fortsetzung der Bundesliga-Berichterstattung ermöglichte. Nach einer zwischenzeitlichen Eskalation der Auseinandersetzungen um die regionale Bundesliga-Berichterstattung verständigten sich DFB und ARD zudem auf ein Stillhalteabkommen, nach dem die ARD im Gegenzug für eine eingeschränkte regionale Fußballberichterstattung in ihren Dritten Programmen vorläufig auf eine Geltendmachung ihres Kurzberichterstattungsrechts verzichtete (epd 10.09.88).

Während die Länder an einem Kurzberichterstattungsrecht arbeiteten, schalteten die Sportverbände das Bundesinnenministerium (BMI) als das Sportministerium des Bundes ein, um eine Beschränkung ihrer Verfügungsrechte zu verhindern. Tatsächlich agierte das BMI als Anwalt der Einnahmeinteressen der Sportverbände, die von der gestiegenen Mediennachfrage profitieren wollten. ${ }^{3}$ Weil die Bundesregierung gegen das Regulierungsvorhaben der Länder nicht nur aus inhaltlichen Erwägungen opponierte, sondern ihnen auch die Kompetenz zur Regelung von Programmbeschaffungsfragen absprach (detailliert: Lauktien 1992), brachte sie jedoch die Eigeninteressen der Länder an der Wahrung ihres Kompetenzbereichs ins Spiel. ${ }^{4}$ Dies führte dazu, dass die Länder trotz Auffassungsdifferenzen über den Umfang des Kurzberichterstattungsrechtes an ihrem Gesetzgebungsvorhaben festhielten, da eine Anerkennung der Einwände der Bundesregierung einen Kompetenzverzicht der Länder impliziert hätte.

Die am 15. März 1990 von den Ministerpräsidenten beschlossene Regelung sah vor, dass allen in Europa zugelassenen Fernsehsendern ein unentgeltliches Kurzberichterstattungsrecht zustand, wobei die Kurzberichte bei kurzfristig und regelmäßig wieder-

3 Diese Position vertrat das BMI auch später vor dem BVerfG, vgl. nur: epd 15.11.97.

4 Die Kompetenzfrage nahm daher auch in dem von den Ländern in Auftrag gegebenen Gutachten zur Kurzberichterstattung eine zentrale Rolle ein, vgl. Lerche/Ulmer (1989). 
kehrenden Veranstaltungen vergleichbarer Art, zu denen die Bundesliga-Begegnungen zu rechnen waren, den Umfang von neunzig Sekunden nicht überschreiten sollten (Art. 10 RStV 1990). Die Bundesregierung strengte daraufhin ein Normenkontrollverfahren gegen das Kurzberichterstattungsrecht an, das aber erst 1998 entschieden wurde.

In der Zwischenzeit zeigte sich, dass der von der Verbandsführung durchgesetzte Verzicht auf die Schaffung einer exklusiven Verwertungshierarchie die Marktchancen der RTLplus-Sendung „Anpfiff“ erheblich geschmälert hatte, so dass der Sender 1991 gänzlich auf eine samstägliche Fußball-Sendung verzichtete. Als die dem Kirch-Konzern zugehörige Internationale Sportrechte Verwertungsgesellschaft GmbH (ISPR) darauf die Bundesliga-Rechte erwarb, bestand sie darum auf einer klaren Verwertungshierarchie zugunsten des Kirch-Senders SAT.1 (SZ 28.03.92).

Das einfachgesetzliche Kurzberichterstattungsrecht erwies sich augrund der besonderen Prozesskonstellationen und des schwelenden Verfassungsrechtsstreits zwar als gerichtlich wenig durchsetzbar. Es begünstigte aber die Entstehung eines entgeltlichen Zweit- und Drittrechtehandels, da es den Nachfragern nach Übertragungsrechten als Drohpunkt für die Erzwingung von Vertragsabschlüssen diente. Der DFB setzte seinerseits seine Machtstellung auf den Programmbeschaffungsmärkten so ein, dass bald kein Fernsehanbieter sein Recht auf unentgeltliche Kurzberichterstattung mehr geltend machte (Petersen 2001). In ähnlicher Weise hielt der DFB im Verhältnis zur ARD die Frage der Existenz von Hörfunkrechten latent. Während die ARD die Existenz solcher Rechte leugnete, stellte sich der DFB auf den Standpunkt, dass die Bundesliga-Verträge mit der ARD auch die Hörfunkberichterstattung beinhalteten.

1998 entschied das Verfassungsgericht den Streit über das Kurzberichterstattungsrecht schließlich in der Kompetenzfrage zugunsten der Länder, inhaltlich formte es aber das Kurzberichterstattungsrecht so aus, dass dieses nun stark den einstigen Überlegungen des Bundes zur Verankerung eines entgeltlichen Zitierrechtes entspricht (BVerfGE 97, 228). Die Frage der Hörfunkrechte wurde dagegen erst im Zuge der Krise des deutschen Digitalfernsehens wieder virulent. 2001 hatte die Kirch-Gruppe die Liga gedrängt, einer Angebotsverknappung im Free-TV und im Live-Hörfunk zuzustimmen, um die Etablierung ihres schwächelnden Pay-TVs zu erleichtern. Die Liga erzwang von der ARD darauf zunächst eine Beschränkung ihrer Live-Hörfunkberichte (Petersen 2001). Nachdem die privaten Veranstalter 2002 einen Musterprozess um die Anerkennung der Hörfunkrechte verloren, entschloss sich die ARD, der unter Einnahmeeinbußen leidenden Liga entgegen zu kommen, die von der ARD ein größeres finanzielles Engagement in der Bundesliga-Berichterstattung forderte. Die ARD erkannte nun die Existenz von Hörfunkrechten an und erwarb diese für einen jährlichen Betrag von rund 5,2 Mio. EUR (kicker 08.04.03).

\subsection{Die Durchsetzung der Schutzlistenregelung auf nationaler und europäischer Ebene}

\subsubsection{Entscheidungsanlässe und-arenen}

Die Digitalisierung des audiovisuellen Sektors warf in den 90er Jahren die Zugangsfrage erneut auf. Dank der verbesserten Realisierbarkeit von Verschlüsselungstechniken eröffnete die Digitalisierung neue Ertragspotenziale, da nun ein beträchtlicher Teil der bisherigen Konsumentenrente im analogen Free-TV abgeschöpft werden konnte (Cameron 1997). Für die folgende Preiseskalation auf dem Sportrechtemarkt war aber auch ausschlaggebend, dass die Marktakteure damit rechneten, dass die erhebliche Fixkostendegression der neuen Technologie einen selbstverstärkenden Entwicklungspfad 
wachsender Erträge zugunsten jenes Unternehmens begründen würde, das über seine Übertragungstechnologie eine dominante Position auf dem digitalen Fernsehmarkt aufbauen würde. Sportrechte waren für die Etablierung der neuen Technologie unentbehrlich, da den Konsumenten nur so ein effektiver Anreiz zur Investition in ein anderes Fernsehsystem gesetzt werden konnte. Diese Strategie erforderte freilich die Verknappung des Angebotes an frei empfangbaren Sportübertragungen über den Erwerb umfangreicher Exklusivpakete (Cowie/Williams 1997; Solberg 2002), so dass die Digitalisierung die Marginalisierungsgefahr für die öffentlichen Fernsehanbieter noch einmal beträchtlich erhöhte.

Die drohende Verknappung des Free-TV-Angebots an attraktiven Sportübertragungen führte zu weiteren medienpolitischen Auseinandersetzungen. Zunächst verschärfte das britische Unterhaus im Februar 1996 eine bereits seit 1954 bestehende Regelungstradition zum Erwerb von Exklusivrechten dahingehend, dass bestimmte Großsportereignisse nicht im Pay-TV ausgestrahlt werden durften. Das Parlament reagierte damit auf ein Angebot Rupert Murdochs an das Internationale Olympische Komitee (IOC), die Exklusivrechte für die Olympischen Spiele der Jahre 2000 bis $2008 \mathrm{zu}$ einem deutlich erhöhten Preis zu erwerben. Der Broadcasting Act von 1996 nahm neben den Olympischen Spielen vor allem Fußballturniere und -begegnungen, Cricketspiele sowie die Finale des Tennisturniers von Wimbledon in die Schutzliste auf (Cowie/Williams 1997). Allerdings erwies sich die Novellierung insoweit als unnötig, als das IOC bei seiner Entscheidung über die Vergabe der Übertragungsrechte dem Einkaufskartell der öffentlichen Fernsehanbieter, der European Broadcasting Union (EBU), den Vorzug gab, um eine breite Empfangbarkeit der Olympischen Spiele zu sichern.

Das britische Vorbild intensivierte sowohl in anderen Mitgliedstaaten als auch auf europäischer Ebene die Diskussion über den Schutz des Zugangs der Öffentlichkeit zu Großsportereignissen. Diese Debatte erhielt neue Brisanz, als der Weltfußballverband FIFA im Juli 1996 zum ersten Mal die globalen Verwertungsrechte an den FußballWeltmeisterschaften 2002 und 2006 für 3,4 Mrd. DM nicht mehr an die EBU, sondern an die Kirch-Gruppe und die Schweizer Marketing-Agentur ISL vergab. Damit reagierte die FIFA auch auf Druck aus den eigenen Reihen. Einige Nationalverbände hatten der FIFA vorgeworfen, die Rechte für die drei vergangenen Weltmeisterschaften zu billig veräußert zu haben. Diese Einwände konnten von der FIFA-Führung kaum ignoriert werden, da diese ihre Machtposition vor allem ihren Bemühungen verdankte, die Kommerzialisierung der Weltmeisterschaften konsequent betrieben und die Einnahmeausschüttungen an die nicht-europäischen Verbände erhöht zu haben (dazu detailliert: Sugden/Tomlinson 1998). Das komplexe Vertragswerk der FIFA mit dem KäuferKonsortium schrieb nur zwingend eine Free-TV-Übertragung für das Eröffnungsspiel, die Halbfinalspiele und die beiden Finalspiele vor (SZ 18.12.97). Das musste bedeuten, dass die Spiele der deutschen Nationalmannschaft nicht mehr für jedermann frei zugänglich sein würden, weil die Kirch-Gruppe diesen Deal nur über eine Verwertung in ihrem überstürzt gegründeten und bislang schwächelnden digitalen Pay-TV refinanzieren können würde (Beck/Prinz 1998). Für die öffentlich-rechtlichen Anstalten drohte mit der wahrscheinlichen Nicht-Übertragung der Fußball-WM 2006 ein dramatischer Bedeutungsverlust, der die Frage der Gebührenlegitimation aufwerfen konnte (vgl. Stolte 2001).

Innerhalb des medienpolitischen Verhandlungssystems der deutschen Bundesländer sollte sich die Forderung nach der Einführung einer nationalen Schutzlistenregelung aufgrund des hohen Konsensbedarfs zunächst als nicht durchsetzbar erweisen. Die bayerische Staatsregierung nutzte ihre Vetoposition und blockierte entsprechende Re- 
gelungsbemühungen, um die wirtschaftlichen Interessen des Kirch-Konzerns zu wahren, der seinen Standort in Bayern hatte (Diesbach 1998). ${ }^{5}$

Während die institutionellen Rahmenbedingungen auf deutscher Ebene für die Verankerung einer Schutzlistenregelung eher ungünstig waren, hatten sie sich auf europäischer Ebene durch die Stärkung des Europaparlaments in den Maastrichter Verträgen entscheidend verbessert. Die Europäische Kommission vertrat seit der Fernsehrichtlinie von 1989 primär einen neo-liberalen Regulierungsansatz im Medienbereich (Gruber 1995; Holznagel 1996) und lehnte eine Schutzlistenregelung als marktfremde „Sonderregelung “ des Fernsehmarktes ab. Innerhalb des Europaparlaments trafen die Marginalisierungsbefürchtungen der öffentlich-rechtlichen Anbieter jedoch vor allem auf die Responsivität von Abgeordneten aus Mitgliedstaaten mit starker „public service“-Tradition. Diese hatten sich bereits seit längerem besorgt über den Liberalisierungskurs gezeigt, der die europäische Medienpolitik seit der Fernsehrichtlinie von 1989 dominierte. Sie verfochten die Aufrechterhaltung eines starken „public service“-Rundfunks nicht zuletzt aus integrationspolitischen Erwägungen (Parrish 2003) und versuchten, ihre Politikvorstellungen in die Novellierung der Fernsehrichtlinie einzubringen, zu der die Kommission im Juli 1995 einen Vorschlag vorgelegt hatte (EG-Amtsbl. vom 10.06.96 C 166). Das Parlament verfügte dabei aufgrund des 1992 eingeführten Mitentscheidungsverfahrens über eine Veto-Position, die sich zu Lasten des Einflusses der Kommission auswirkte. Bis dahin hatte die Kommission das alleinige formelle Initiativrecht für den Entwurf europäischer Sekundärrechte inne, nun war das Parlament in der Lage, im Mitentscheidungsverfahren selbst Politikinitiativen durchzusetzen (generell: Pollack 1997).

\subsubsection{Interaktionsverläufe und-ergebnisse}

Wie bei der Verankerung des nationalen Kurzberichterstattungsrechts wurden die Policy-Präferenzen einzelner politischer Akteure zugunsten eines „public service“-Rundfunks aber erst durchsetzungsfähig, als die Entwicklung des Marktes für Sportübertragungsrechte die Gefahr einer Angebotsverknappung im Free-TV konkret werden ließ und zu einer erheblichen Mobilisierung der öffentlichen Meinung führte. Die intensive öffentliche Debatte um den Zugang zu den Begegnungen der Fußball-Weltmeisterschaft schuf wiederum ein Gelegenheitsfenster, das es den Verfechtern einer „public service“Idee im Europaparlament erlaubte, bei der Beratung der Novelle der Fernsehrichtlinie eine Schutzlistenregelung durchzusetzen. Um ihr Vorhaben auch in der Fraktion der Europäischen Volkspartei mehrheitsfähig zu machen, die eher dem Liberalisierungsansatz der Kommission zuneigte, appellierten die Verfechter einer „public service“-Konzeption auch an die Eigeninteressen des wenig sichtbaren Europaparlaments, das auf diese Weise gegenüber seiner Wählerschaft eine öffentlichkeitswirksame Legitimation erlangen konnte. ${ }^{6}$ Der Entwurf des Parlaments verlangte jedoch nicht nur eine obligatorische Liste, sondern ließ den Mitgliedstaaten wenig Spielraum, die Frage eines breiten öffentlichen Zugangs zu solchen Ereignissen durch andere Maßnahmen zu sichern (EG-Amtsbl. vom 01.12.96 C 362/60). Schließlich setzte die Kommission zusam-

$5 \mathrm{Zu}$ den Beziehungen zwischen dem Kirch-Konzern und der bayerischen Staatsregierung, vgl. Gehring (2003).

6 Diese Interpretation beruht vor allem auf der Darstellung beteiligter Akteure.

Inzwischen hatten UEFA und DFB offen die Vermarktungspolitik des Weltverbandes kritisiert (FAZ 03.11.97; SZ 03.11.97). 
men mit dem Ministerrat im folgenden Vermittlungsverfahren durch, dass der Erlass einer Schutzlistenregelung nach der Fernsehrichtlinie vom Juni 1997 in das Belieben der Mitgliedstaaten gestellt wurde (EG-Amtsbl. vom 30.07.97 L 202; Diesbach 1998).

Damit hatten sich auch die politischen Rahmenbedingungen für die parallel in Deutschland heftig geführte Diskussion um eine nationale Schutzlistenregelung verändert. Die bayerische Regierung hatte die Verabschiedung einer deutschen Schutzlistenregelung unter Hinweis auf die verfassungsrechtlichen Risiken eines solchen gesetzgeberischen Eingriffes in die Rechtsposition der Kirch-Gruppe blockiert. Sie versuchte zunächst, eine einvernehmliche Lösung des Zugangsproblems unter Einbeziehung der Kirch-Gruppe, der Sportverbände und der öffentlich-rechtlichen Anbieter zu forcieren. Dieses Vorhaben scheiterte einerseits am Widerstand der Kirch-Gruppe, die sich weiterhin nur den Mindestanforderungen der FIFA beugen wollte (FK 24.10.97). Andererseits bestanden die öffentlich-rechtlichen Anstalten auf einer gesetzlichen Regelung (epd 11.10.97). Aufgrund der öffentlichen Kontroversen und des drohenden Verlustes der politischen Unterstützung rückte inzwischen auch die FIFA von ihrer Strategie der Einnahmemaximierung ab. Der Weltfußballverband bekannte sich zur breiten Zugänglichkeit der WM und versicherte, dass er nicht gegen nationale Schutzlistenregelungen vorgehen würde (SZ 04.12.97). Damit waren entscheidende rechtliche Unsicherheiten für die Verabschiedung einer deutschen Listenregelung beseitigt worden. Angesichts des enormen öffentlichen und politischen Drucks auf die Landesregierungen gab daher auch Bayern seinen Widerstand gegen eine Schutzlistenregelung auf (SZ 02.12.97), so dass die Ministerpräsidenten am 18. Dezember 1997 die Vorarbeiten für eine verbindliche Schutzlistenregelung aufnahmen. Am 18. März 1998 fasste die Ministerpräsidentenkonferenz den Beschluss, eine Schutzlistenregelung als $\ 5$ a im Zuge der vierten Änderung des Rundfunkstaatsvertrages einzuführen, die für bestimmte Ereignisse eine Verwertung im Pay-TV verbot. Diese Liste umfasst außer den Olympischen Spielen alle Auftritte der deutschen Fußballnationalelf im In- und Ausland einschließlich der Welt- und Europameisterschaften sowie die Eröffnungs-, Halbfinal- und Finalspiele dieser Turniere und das Finale des DFB-Pokals (Diesbach 1998).

\subsubsection{Die politische Stabilisierung des Sportrechtemarktes}

Mit der Schutzlistenregelung hatten die öffentlich-rechtlichen Anbieter ein zweites Mal eine erhebliche Einschränkung der Verwertungsmöglichkeiten von Sportrechteinhabern durchgesetzt. Sportrechte waren politisch als ein Gut definiert worden, über das die Rechteinhaber aufgrund der öffentlichen Bedeutung attraktiver Sportereignisse nur eingeschränkt verfügen können. Allerdings sicherte dieser Erfolg dem öffentlich-rechtlichen Rundfunk nur, dass er bei der künftigen Vergabe von Übertragungsrechten an Großsportereignissen als Free-TV-Anbieter überhaupt in Betracht gezogen wurde. Sehr viel wahrscheinlicher erschien es, dass werbefinanzierte Free-TV-Anbieter aufgrund ihrer höheren Finanzierungspotenziale von der Schutzlistenregelung profitieren würden (Beck/Prinz 1998).

Tatsächlich führte die Schutzlistenregelung jedoch zu einem Vertragsabschluss der öffentlich-rechtlichen Anstalten mit dem Kirch-Konzern. Die Schutzlistenregelung hatte die Verwertungsmöglichkeiten der Kirch-Gruppe auf dem deutschen Markt erheblich eingeschränkt und drohte die Refinanzierbarkeit des Rechteerwerbs für die WM 2002 in Korea und Japan zu gefährden, da die ungünstigen Übertragungszeiten eine Verwertung im werbefinanzierten Free-TV nicht sonderlich lohnend erscheinen ließen. Die Kirch-Gruppe befand sich zu diesem Zeitpunkt bereits in einer wirtschaftlichen Krise, 
da sich die Etablierung des digitalen Pay-TV schwieriger als erwartet gestaltete (Gehring 2003). Der Konzern nahm daher Verhandlungen mit den öffentlich-rechtlichen Fernsehanstalten auf, musste einen möglichen Vertrag über die Übertragungsrechte an der WM 2002 aber mit einer Option auf die Übertragungsrechte an der in Deutschland stattfindenden WM 2006 verbinden, deren Übertragung ARD und ZDF zentrale strategische Bedeutung zumaßen. Die Verhandlungen scheiterten jedoch, weil die Kirch-Gruppe keine bindenden Zusagen zur Fußball-WM im Jahr 2006 machen wollte. In der Folge wurden die Anstalten jedoch politisch zu einem Vertragsabschluss gedrängt. Dabei engagierte sich nicht nur die bayerische Landesregierung aufgrund ihrer Standortinteressen, sondern auch sozialdemokratische Medienpolitiker, die neben der Sorge vor einem weiteren Bedeutungsverlust des öffentlich-rechtlichen Fernsehens vor allem Furcht vor einem Marktzutritt Rupert Murdochs hatten, der sich bereits finanziell an der KirchGruppe beteiligt hatte. Der erhebliche Einfluss, den der Konservative Murdoch auf die politische Meinungsbildung in Großbritannien ausübte, reaktivierte sozialdemokratische Ängste über eine mögliche Benachteiligung durch kommerzielle Medienanbieter (vgl. FK 09.03.01). ${ }^{7}$ Schließlich erwarben die Anstalten die Senderechte an der Fußballweltmeisterschaft $2002 \mathrm{zu}$ einem enormen Preis und unter großen Zugeständnissen an den Kirch-Konzern beim Zugang zu anderen Sportgroßereignissen. Für die WM 2006 erhielten sie eine Option für den Rechteerwerb, die an die Zahlung eines Mindestpreises gebunden wurde, so dass sich die Kirch-Gruppe die Möglichkeit offen hielt, die WM-Rechte für einen höheren Preis an andere Bieter zu verkaufen (Ott 2001). Allerdings konnten die Vertragsparteien keine Einigung über die von der Kirch-Gruppe von Anfang an geforderte Verschlüsselung der Satellitenübertragung erreichen, die der Konzern für notwendig hielt, um die Vermarktung der Pay-TV-Rechte im europäischen Ausland nicht zu gefährden (vgl. FAZ 21.01.01). Dies führte dazu, dass die Anstalten 2002 auf eine Übertragung der Fußball-WM in ihren digitalen Satellitenangeboten verzichteten, um gerichtliche Auseinandersetzungen mit den ausländischen Vertragspartnern der Kirch-Gruppe zu vermeiden (SZ 02.08.02).

\subsection{Die wettbewerbsrechtliche Behandlung von Kollektivvermarktungssystemen}

\subsubsection{Entscheidungsanlässe und-arenen}

Während die Einschränkungen der Verfügungsrechte der Rechteinhaber zugunsten der Zugangsinteressen der Allgemeinheit „nur“ das Einnahmepotenzial von Sportverbänden und Profiligen reduzierten, erschütterten die wettbewerbsrechtlichen Interventionen gegen die Zentral- und Kollektivvermarktungsregime die Grundfesten des europäischen Sportmodells. Auch hierbei handelte es sich um ein Regulierungsproblem, das sich erst infolge der Liberalisierung der europäischen Fernsehmärkte stellte, da sich bis zur Deregulierung des audiovisuellen Sektors öffentliche Fernsehanbieter und Sportverbände als bilaterale Monopole in einem relativen Machtgleichgewicht gegenübergestanden hatten.

Im Zuge ihrer Marktöffnungspolitik nach der Deregulierung der Fernsehmärkte verhinderten die Wettbewerbsbehörden zunächst, dass die öffentlichen Anbieter ihre noch

$7 \mathrm{Zu}$ diesen Befürchtungen gegenüber einem Marktzutritt Murdochs oder Berlusconis, vgl. u. a. DER SPIEGEL 08.04.02; FR 08.04.02, FR 09.04.02. 
bestehende Nachfragemacht einsetzten, um der privaten Konkurrenz den Marktzutritt zu erschweren. Das Bundeskartellamt untersagte 1987 den von ARD und ZDF mit dem Deutschen Sportbund geschlossenen Globalvertrag, mit dem sich die Anstalten den exklusiven Zugriff auf die Übertragung von sieben der zehn beliebtesten Sportarten gesichert hatten (WuW/E BKartA 1987, 2273). Die Wettbewerbsdirektion der EU-Kommission ging dagegen seit 1987 auf Beschwerden privater Anbieter gegen das öffentlichrechtliche Einkaufskartell der EBU vor, das sich weigerte, Unterlizenzen für die von ihm exklusiv erworbenen Übertragungsrechte an private Veranstalter zu vergeben. Obwohl die EBU ihre Regeln für den Erwerb von Unterlizenzen 1993 überarbeitete, halten die Auseinandersetzungen über die Vereinbarkeit dieses öffentlich-rechtlichen Einkaufskartells mit dem europäischen Wettbewerbsrechts bis heute an. ${ }^{8}$

Nach diesen marktöffnenden Interventionen stellte sich jedoch bald die Frage, ob das Angebotsverhalten der Sportverbände nicht neue marktkorrigierende Eingriffe der Wettbewerbsbehörden notwendig machen würde. Die Sportverbände nutzten die gestiegene Nachfrage nach attraktiven Übertragungsrechten, um gegenüber den Nachfragern ihr Angebotsmonopol offensiver zur Geltung zu bringen. Das Engagement der Wettbewerbsbehörden hatte zur Folge, dass die Frage der Notwendigkeit von Kartellbildungen im Sport und damit der Stellung der Sportverbände in der Verwertungskette des Profisports umfassend auf die politische Agenda geriet. Obwohl das deutsche Kartellrecht verschiedene Möglichkeiten gekannt hätte, das Zentralvermarktungssystem nicht als wettbewerbsrechtswidriges Kartell zu behandeln, ${ }^{9}$ vertraten die Wettbewerbsbehörden eine orthodoxe wettbewerbsrechtliche Position. Sie bezweifelten die Notwendigkeit der sportspezifischen Kartellbildungen zur Erreichung der Ziele der sportlichen Ausgeglichenheit und der Finanzierung von Solidaritätszahlungen an den Amateurbereich (Parlasca 1993; Parlasca/Szymanski 2002). Die Indifferenz der Wettbewerbsbehörden gegenüber den vermeintlichen Besonderheiten des Sports führte dazu, dass sich vor allem das europäische Wettbewerbsrecht als flexible Waffe für Akteure anbot, die sich durch Regelungen der Sportverbände benachteiligt sahen (Weatherill 2003).

Auf nationaler Ebene bot sich für sportpolitische Anliegen immerhin die Möglichkeit, eine Intervention des Bundeskartellamtes durch die Schaffung einer gesetzlichen Ausnahmeregelung im Gesetz gegen Wettbewerbsbeschränkungen zu revidieren. Ein solches Vorhaben erforderte jedoch eine doppelte Mehrheit zugunsten sportpolitischer Erwägungen in Bundestag und Bundesrat gegen die Bedenken der Wettbewerbspolitiker. Wie zu zeigen sein wird, gelang diese Mehrheitsbildung nur aufgrund eines außerordentlich günstigen Gelegenheitsfensters.

Die institutionellen Rahmenbedingungen für eine sportpolitische „Korrektur“ wettbewerbsrechtlicher Interventionen der Wettbewerbsdirektion der Europäischen Kommission waren noch weitaus ungünstiger. Die Konstitutionalisierung des europäischen Wettbewerbsrechts durch den Europäischen Gerichtshof hat einen starken Deregulierungsbias der supranationalen Politik zur Folge und privilegiert eine Politik der negati-

8 Die EBU erwirkte zwar 1993 und 2000 positive Freistellungserklärungen der EU-Kommission, das Gericht erster Instanz verwarf diese Entscheidungen zuletzt am 17. Oktober 2002 jedoch mit dem Argument, dass die Unterlizenzregelungen den Konkurrenten der Mitglieder der EBU keinen ausreichenden Zugang zu den Rechten für die Übertragung sportlicher Ereignisse garantierten (Rs. T 185-00).

9 Der DFB brachte in seinen Einlassungen u. a. die Immanenztheorie und den Arbeitsgemeinschaftsgedanken vor, vgl. Stopper 1997; Waldhauser 1999. 
ven Integration, die einseitig auf die marktschaffende Beseitigung von Handelshindernissen abzielt. Die Europäische Kommission nutzt ihre weit reichenden Befugnisse als europäische Wettbewerbsbehörde als flexibles Instrument zur Erreichung ihrer binnenmarktpolitischen Integrationsziele (Schmidt 1998). Sie befindet sich dabei in einer starken strategischen Position, weil sich die Mitgliedstaaten einstimmig auf eine Vertragsrevision verständigen müssen, wenn sie weitere Liberalisierungsbemühungen der Kommission verhindern wollen (Scharpf 1999).

\subsubsection{Interaktionsverläufe und-ergebnisse im nationalen Kontext}

Der DFB hatte sein Angebotsmonopol auf dem deutschen Fernsehmarkt zwar diszipliniert und disziplinierend eingesetzt, indem er durch eine breite Rechtestreuung verhinderte, dass die großen Fernsehanbieter selbst ein Kartellrechtsverfahren einleiteten (explizit: epd 07.02.96). Als der DFB jedoch 1989 das Zentralvermarktungsregime auf die bis dahin von den Vereinen vermarkteten Heimspiele im UEFA-Pokal ausdehnte und das sportliche Risiko über das Ausscheiden der Clubs auf die Rechteverwerter abwälzte, nutzte das Bundeskartellamt 1993 sein Aufgriffsermessen und ging gegen diesen vermeintlichen Kartellbeschluss vor. Dabei versuchte das Kartellamt von Anfang an, eine Präzedenzentscheidung auch für die Zentralvermarktung der Bundesliga herbeizuführen. Das BKartA identifizierte die Clubs als eigentliche Veranstalter und damit Inhaber der Übertragungsrechte (Stockmann 1996).

Diese wettbewerbsrechtlich motivierte Verschiebung der Verfügungsrechte zugunsten der Clubs bedeutete letztlich, dass die Sportverbände ihr Veranstaltungsmonopol und damit die Kontrolle über den Profibereich zu verlieren drohten. Diese Entwicklung gefährdete die Integrität des europäischen Sportmodells, da nun sogar die kommerzielle Übernahme des Profifußballs möglich geworden war. Die Sezessionsanreize für die Proficlubs waren enorm und hatten in England bereits 1992 dazu geführt, dass die erste Spielklasse - animiert durch eine Offerte des im Besitz von Rupert Murdoch befindlichen BSkyB und der englischen Football Association, die sich in Konkurrenz zum LigaVerband befand, - die Solidargemeinschaft mit den unteren Spielklassen aufkündigte und die Premier League gründete (Conn 1999). Die digitale Übertragungstechnik verstärkte aber auch die internen Verteilungskämpfe in den Profiligen, da sie den Spitzenvereinen die Perspektive bot, über die verschlüsselte Übertragung ihrer Begegnungen im Payper-View direkt von ihrem überlegenen Ertragspotenzial zu profitieren. So revoltierten die niederländischen Spitzenvereine gegen den niederländischen Fußballverband KNVB, der sich 1996 glücklos an der Pay-TV-Verwertung der Profiliga-Spiele versuchte. Sie wehrten sich gerichtlich mit Erfolg gegen die Satzungsänderung des Verbandes, mit der dieser sich nachträglich die Vermarktungsrechte zugeschrieben hatte. Damit führten die Spitzenvereine die Erosion des Zentralvermarktungssystems herbei (Waldhauser 1999).

Für den DFB war diese Entwicklung äußerst beunruhigend. Der Verband fühlte sich traditionell in besonderem Maße dem Amateurismus verpflichtet und hatte sich stets um die Kontrolle und institutionelle Einbettung des Profifußballs bemüht (Eisenberg 1997). Diese Kontrolle war durch die Verschiebung der Verfügungsrechte zugunsten der Clubs ernsthaft gefährdet. Darüber hinaus hielt die DFB-Führung die Aufrechterhaltung der Zentralvermarktung für die sportliche Ausgeglichenheit der Bundesliga für notwendig. Der DFB ging daher gegen die Kartellamtsentscheidung von 1994, die die Zentralvermarktung der UEFA-Pokal-Heimspiele untersagt und die Clubs zu den Rechteinhabern erklärt hatte (WuW 1995, BKartA 2682-700), gerichtlich vor und wurde vor der erwarteten letztinstanzlichen Entscheidung des Bundesgerichtshofs 1997 auch politisch aktiv 
(SZ 03.11.97). Sein Interesse an der Fortführung der Zentralvermarktung entsprach zum damaligen Zeitpunkt auch dem der Mehrheit der Profivereine, die für den Fall der Beseitigung des Zentralvermarktungssystems um ihre wirtschaftliche Zukunft bangten. ${ }^{10}$

Die Verbandsführung fand dabei auf Ebene des deutschen Föderalismus Zugang zum politischen Prozess. Auf Seiten der Sportpolitik wurde die Unterstützung zwischen Politikern und den Funktionären der Sportverbände durch das gemeinsame Interesse an der Aufrechterhaltung des europäischen Sportmodells mit seiner Verknüpfung von Profi- und Amateurbereich und vertikalen Solidarmechanismen gewährleistet (vgl. epd 22.11.97). ${ }^{11}$ Zudem fielen die Auseinandersetzungen über die Zentralvermarktungsregime in die Diskussion über das Bosman-Urteil des EuGH, wobei die Liberalisierung des Spielermarktes von großen Teilen der nationalen Sportpolitik aufgrund der Verstärkung des Kommerzialisierungstrends und der Gefährdung der Nachwuchsausbildung bereits als Bedrohung des europäischen Sportmodells wahrgenommen wurde (Parrish 2003). Dabei verbanden die Länder mit dem Zentralvermarktungssystem insoweit auch eigene sportpolitische Standortinteressen, als dass die Finanzierung vor allem traditionsreicher Zweit-Liga-Clubs gefährdet schien (FAZ 20.12.97), die für die regionale Identifikationen in den Ländern eine zentrale Rolle spielen. Für die medienpolitische Bewertung der Zentralvermarktungsfrage waren einerseits die bisherigen Einschränkungen der Verfügungsrechte der Sportrechteinhaber entscheidend. Andererseits wollten zentrale Akteure im medienpolitischen Verhandlungssystem der Länder an der Zentralvermarktung festhalten, um einem deutschen Digitalfernsehen die Durchsetzung nicht weiter zu erschweren. Schließlich traten die Fernsehanbieter in der Debatte um die Zentralvermarktung als primär betroffene Marktgegenseite nicht in Erscheinung, weil sie dem DFB aufgrund der Politik der breiten Rechtestreuung verpflichtet waren oder wie die dominierenden privaten Medienkonzerne Bertelsmann und Kirch, die ihre Pay-TV-Aktivitäten zum damaligen Zeitpunkt verschmolzen hatten (FAZ 03.07.97), ein eigenes Interesse an der Aufrechterhaltung der Zentralvermarktung verfolgten.

Auf bundespolitischer Ebene profitierte der DFB aber vor allem davon, dass die Entscheidung des BGH in ein doppelt günstiges Gelegenheitsfenster fiel, nämlich in die Endberatungen des Gesetzes gegen Wettbewerbsbeschränkungen (GWB) und in die Vorphase des Bundestagswahlkampfes 1998. Wie aus den Beratungsunterlagen des Bundestages hervorgeht, stimmte das Parlament der vom Bundesrat in seiner Stellungnahme zum Entwurf der GWB-Novelle erhobenen Forderung nach einer gesetzlichen Ausnahmeregelung für die Zentralvermarktung im GWB schließlich im Mai 1998 zu, weil die Bundesparteien ein gemeinsames Interesse daran hatten, das riskante und unkalkulierbare Thema nicht auf die Agenda des Bundestagswahlkampfes geraten zu lassen (Meier 2004).

10 Bemerkenswerterweise hielten sich die Spitzenvereine während der Auseinandersetzung um die GWB-Novellierung weitgehend zurück. Diese Zurückhaltung konnte im Rahmen des Forschungsprojektes nicht hinreichend erklärt werden, da die Spitzenvereine später erheblichen Druck auf die Sportverbände ausübten.

11 Die folgende Darstellung zur komplexen Motivlage der Bundesländer basiert vor allem auf Interviews mit den Rundfunkreferenten, vgl. auch: die Begründung in BRDrs. 852/2/97 sowie das Interview von Ministerpräsident Kurt Beck im DFB-Journal 2/99. 


\subsubsection{Interaktionsverläufe und-ergebnisse auf europäischer Ebene}

Während der DFB damit auf nationaler Ebene in der Zentralvermarktungsfrage politisch sehr erfolgreich agiert hatte, riefen seine Bemühungen um eine Ausnahmeregelung die Wettbewerbsdirektion (GD 4) der Europäischen Kommission auf den Plan. Diese versuchte zunächst, die Schaffung einer deutschen Ausnahmeregelung zu verhindern (FAZ 31.03.98). Die Kommission hatte sich zuvor bereits mit den 1988 verschärften Ausstrahlungsregelungen der UEFA befasst, die die grenzüberschreitende Verbreitung von Sportübertragungen generell verboten, und die UEFA 1993 zu einer ersten Liberalisierung dieser Verknappungspraktiken gezwungen (EG-Amtsbl. vom 26.06.01 L 171). Zum damaligen Zeitpunkt widmete sich die GD 4 im Sportbereich vor allem den Vermarktungspraktiken des internationalen Motorsportverbandes FIA, der sein Zentralvermarktungsregime 1996 auf alle internationalen Motorsport-Serien ausgedehnt hatte, um Angebotsverknappungen und Preissteigerungen zugunsten der Vermarktung der Formel 1 durchzusetzen. Bei der Kommission waren eine Reihe anonymer Beschwerden gegen die FIA eingegangen (Van Miert 2000).

Darüber hinaus warf die Zentralvermarktung der von der UEFA 1992 werbewirksam aufgebauten Champions League erhebliche wettbewerbsrechtliche Bedenken auf. Die UEFA hatte sich für diesen Wettbewerb auf einer außerordentlichen Mitgliederversammlung die Vermarktungsrechte an einem Großteil der Spiele abtreten lassen und zwang einzelne Vereine zum Bruch bestehender Vermarktungsverträge. Die UEFA verknappte zudem das Angebot an Live-Übertragungen künstlich und war durch ihre Alleinanbieterstellung in der Lage, Monopolpreise durchzusetzen. Die Rechte wurden zudem im Paket exklusiv für bis zu vier Jahre an einen einzigen Rundfunkanbieter je Mitgliedstaat veräußert. Dabei konnte der Rechteverwerter alle weiteren Verwertungsmöglichkeiten an den Champions League-Spielen im Pay- oder Free-TV ausschöpfen oder ggf. nicht nutzen, um seinen Exklusivitätsanspruch nicht zu gefährden. Schließlich gab die UEFA keinesfalls die gesamten Erlöse an die mitwirkenden Vereine und Verbände weiter, sondern behielt selbst einen erheblichen Anteil von $25 \%$ für eigene Aufgaben ein (Hellenthal 2000).

Mit der Verlagerung der wettbewerbspolitischen Auseinandersetzungen auf die europäische Ebene hatten sich die institutionellen Parameter des Entscheidungsprozesses stark zu Ungunsten der Sportverbände geändert. Obwohl die Mitgliedstaaten versuchten, die Kommission von ihrem orthodoxen Liberalisierungskurs im Sportbereich abzubringen, identifizierte diese die Zentral- und Kollektivvermarktungssysteme als Wettbewerbsbeschränkungen (EU-Kommission 1998b). Ihre kritische Position veränderte entscheidend die Machtkonstellationen im Profifußball, da die schwelende kartellrechtliche Auseinandersetzung die Drohposition der Spitzenvereine gegenüber den Sportverbänden und den ertragsschwächeren Clubs stärkte. Zwischenzeitlich ließen die Auseinandersetzungen zwischen Spitzenclubs und Sportverbänden sogar ein „natürliches Ende“ der Vermarktungskartelle erwarten. Die Konflikte innerhalb des organisierten Fußballs sollten jedoch nicht im Sinne der Wettbewerbsbehörden durch ein Zerbrechen der Vermarktungskartelle, sondern durch eine ungleichere Verteilung der Einnahmen gelöst werden. Zunächst erreichten die europäischen Spitzenvereine eine Veränderung des Wettbewerbsdesigns der Champions League, nachdem die Vermarktungsagentur Media Partners International Limited (MPI) ihnen Anfang 1998 das Angebot einer geschlossenen europäischen Superliga unterbreitet hatte. Um die sezessionswilligen Clubs von einer Abwanderung abzuhalten, sah sich die UEFA gezwungen, das Wettbewerbsdesign und die Vermarktung der Champions League Ende 1998 zu- 
gunsten der Spitzenclubs aus den wichtigsten Profiligen zu überarbeiten. Dies gelang freilich nur, weil sich der Markt für Sportrechte in einer Wachstumsphase befand und sich die Einnahmen der UEFA verdoppeln ließen (Hoehn/Szymanski 1999).

In ähnlicher Weise vollzog sich die Entsolidarisierung der Bundesliga-Vermarktung. Der DFB bzw. die von ihm kontrollierte Liga-Führung hatte nach der GWB-Novellierung auf Druck der EU-Kommission einen Freistellungsantrag für die Zentralvermarktung der Bundesliga gestellt. In dem Antrag hatte der Verband reklamiert, zumindest Miteigentümer der Übertragungsrechte zu sein. Dies traf auf den Widerstand der Spitzenvereine (FAZ 25.01.99). Dem DFB wurde dabei zum Verhängnis, dass er den Solidarausgleich mit einer restriktiven Vermarktungspolitik, vor allem im Pay-TV, verknüpft hatte. Dank der eskalierenden Nachfrage auf dem Sportrechtemarkt war eine Konstellation eingetreten, in der die Spitzenvereine dem Rest der Liga eine Revision des Vermarktungsarrangements in Aussicht stellen konnten, nach der den kleinen Vereinen zumindest ihre bisherigen Einnahmen garantiert wurden, die Einnahmezuwächse aber stärker den Spitzenvereinen zu Gute kommen sollten (Zacharias 1999, S. 147-8). Der Konflikt zwischen DFB-Führung und Spitzenvereinen wurde schließlich dadurch gelöst, dass das Zentralvermarktungssystem aufrechterhalten, der Verteilungsschlüssel jedoch leistungsabhängiger ausgestaltet wurde. Möglich wurde diese Konfliktlösung, weil der DFB den Kirch-Konzern mit der Drohung eines Vertragsverzichtes zu einer Verdopplung des Kaufpreises für die Bundesligarechte zwingen konnte, da der Konzern mit seinem eigenständigen Decodersystem bereits erhebliche „sunk costs“ getätigt hatte und sein Digitalangebot ohne exklusive Sportangebote kaum überlebensfähig war (FAZ 27.09.99). Allerdings leiteten die Bundesliga-Vereine in Reaktion auf die Auseinandersetzungen über die Vermarktungspolitik des DFB die rechtliche Verselbstständigung der Liga ein, was von Verbandsführung und Sportpolitik gleichermaßen als partielle Abkehr vom klassischen Sportmodell verstanden wurde (Meier 2004).

Die Sportpolitiker der Mitgliedstaaten brachten gegenüber der EU-Kommission ihre Bedenken gegen eine mögliche „Amerikanisierung“ des europäischen Sportmodells zum Ausdruck (Parrish 2003). Die schwierige Revision des europäischen Vertragswerks erlaubte es der Kommission jedoch zunächst, ihren wettbewerbsrechtlichen Liberalisierungsansatz weiter zu verfolgen. Die Mitgliedstaaten waren in der Frage einer Vertragsrevision nicht konsensfähig, weil vor allem die britische Regierung für den Fall einer Erwähnung des Sports im europäischen Vertragswerk eine weitere Kompetenzerosion befürchtete (Schneider 2002). Die Mitgliedstaaten forderten die Kommission daher auf ihrem Wiener Gipfel im Dezember 1998 lediglich auf, einen Bericht über die Bewahrung der traditionellen Sportstrukturen in Europa vorzulegen. Die Kommission war daraufhin aber nur zu marginalen Zugeständnissen bereit (vgl. IP/99/133).

Der Widerstand der Mitgliedstaaten gegen den Liberalisierungsansatz der Kommission im Sport nahm jedoch beträchtlich zu, als die Kommission mit der vollständigen Abschaffung des Transfersystems und damit der für das europäische Sportmodell charakteristischen Ausbildungsentschädigungen an den Amateurbereich drohte, die sie als wettbewerbsbeschränkende Arrangements zur Ausbeutung der Spieler wahrnahm (Egger/Stix-Hackl 2002). Die Regierungschefs verlangten von der Kommission auf dem Nizzaer Gipfel im Dezember 2000 daher einerseits eine kooperative Lösung des Streits über das Transfersystem und forderten sie bei dieser Gelegenheit gleichzeitig zur Aufrechterhaltung von Solidaritätsmechanismen im Sport auf (Schneider 2002). Statt sich auf eine Vertragsrevision zu einigen, zwangen die Mitgliedstaaten die Kommission im Frühjahr 2001 zur Aufrechterhaltung einer Reihe von Sonderregulierungen auf dem Spielermarkt durch die Drohung einer direkten Einigung zwischen den Regierungschefs 
und den Fußballverbänden (Egger/Stix-Hackl 2002; Dabscheck 2003). Nach diesem erzwungenen Transferkompromiss agierte die Kommission auch in weiteren Sportfällen erkennbar pragmatischer und kompromissbereiter (Parrish 2003; Weatherill 2003). Dieser Trend schlug sich auch bei der Behandlung der Zentralvermarktungsfrage nieder. Allerdings kam der Kommission entgegen, dass die Vermarktungssysteme der europäischen Profiligen sehr unterschiedlich ausgestaltet sind (Stopper 1997), so dass der Widerstand der Mitgliedstaaten gegen die Beseitigung von Kollektivvermarktungssystemen weniger konzertiert ausfiel als in der Transferfrage. Zudem waren die Politikpräferenzen der Mitgliedstaaten in der Zentralvermarktungsfrage komplexer als im Hinblick auf die Liberalisierung des Spielermarktes. Die Kommission versuchte davon zu profitieren, dass die Verknappungspraktiken der Sportverbände dem Projekt der Mitgliedstaaten zur Belebung der neuen Medienmärkte widersprachen. Auf dieses Projekt hatten sich die EU-Mitgliedstaaten auf dem Gipfel von Sevilla im Juni 2002 verständigt, um Europa zum wettbewerbsintensivsten, wissensbasierten Wirtschaftsraum zu machen (COM [2002] final). Die Kommission ordnete ihre Liberalisierungsbemühungen auf dem Sportrechtemarkt in den Kontext dieses Politikvorhabens. Sie warf den Fußballverbänden primär vor, dass die exklusive Paketvergabe dazu geführt habe, dass neue Medienanbieter im Internet- oder UMTS-Bereich keine Übertragungsrechte erwerben konnten, weil die Exklusiverwerber kein Interesse an der Stärkung dieser neuen Konkurrenz hätten. Damit legte sie ihren Politikschwerpunkt auf die Minimierung der negativen Auswirkungen der Vermarktungspraktiken im Profisport auf die nachgelagerten Medienmärkte. ${ }^{12}$ Gleichzeitig weigerte sich die Kommission aber, das Solidaritätsargument zur Basis einer wettbewerbsrechtlichen Sonderbehandlung des Profifußballs zu machen. Damit blieb nicht nur der prinzipielle Konflikt mit den sportpolitischen Überzeugungen ungelöst, vielmehr hielt sich die Kommission auf diese Weise die Option für eine weitere Liberalisierung des Sportrechtemarktes offen (vgl. Schaub 2002; Ungerer 2003). Dabei kam ihr entgegen, dass sich nach dem Platzen der New EconomyBlase eine Abkühlung der Preiseskalation auf dem Sportrechtemarkt abzeichnete. Inzwischen hatten sich die Erwartungen in das Ertragspotenzial und die strategische Relevanz von Sportrechten als übersteigert erwiesen (Berg/Rott 2000). Dies erleichterte sowohl der UEFA als auch dem DFB eine Überarbeitung ihrer Vermarktungspolitiken, da die Nachfrager nach Übertragungsrechten nicht mehr auf die Aufrechterhaltung einer umfassenden Paketvermarktung drängten.

Allerdings sah sich die UEFA gezwungen, um die Unterstützung der in der G-14 organisierten europäischen Spitzenvereine zu werben, damit diese die Freistellung der Zentralvermarktung der Champions League nicht torpedierten. Durch diese Einbeziehung der G-14 erreichte die Kommission ihr Ziel einer Intensivierung des Wettbewerbs auf dem Sportrechtemarkt. Die UEFA musste auf Druck der G-14 in den neuen Medien eine begrenzte dezentrale Vermarktung sowie eine Rechte-Mitinhaberschaft der Proficlubs an den Übertragungsrechten anerkennen (FAZ 28.01.02). Im Ergebnis akzeptierte die Kommission zwar die weit gehende Aufrechterhaltung der Zentralvermarktung der Champions League durch die UEFA einschließlich der Exklusivverwertung auf dem gegenwärtig noch bedeutendsten Rechtemarkt im Free-TV und im Pay-TV. Die

12 Dieser begrenzte Regulierungsfokus ist auch für die Position der Kommission gegenüber der Rechtevergabe an den Olympischen Spielen 2004 in Griechenland kennzeichnend, auch hier erklärte die Wettbewerbsdirektion, sich darauf konzentrieren zu wollen, den Markt für Internetund UMTS-Anbieter über attraktive Sportrechte zu beleben (Monti 2003). 
GD 4 zwang die UEFA jedoch zu einer Aufteilung ihrer Rechte in verschiedene Pakete und setzte eine Durchbrechung des Zentralvermarktungssystems und Exklusivitätsprinzips bei Hörfunkübertragungen, Internet- und UMTS-Angeboten durch. Hier können die Clubs ihre Rechte nun auch individuell verwerten. Zudem fallen von der UEFA nicht-vermarktete Rechte in die Verfügungsgewalt der Clubs zurück. Schließlich forderte die Kommission für die Rechtevergabe ein transparentes und faires Verfahren (IP/02/806; EG-Amtsbl. vom 17.08.2002 C 196). Damit verzichtete die Kommission zunächst auf eine umfassende Liberalisierung des Profifußballs, jedoch stärken die „fall back"-Klauseln zugunsten der Clubs die zentrifugalen Kräfte im europäischen Profifußball. Daneben führt das neue Zentralvermarktungsregime der UEFA weder zwingend zu einer Aufteilung der Rechte auf mehrere nationale Rundfunkveranstalter noch zu einer Live-Übertragung im Free-TV.

Das neue Vermarktungsmodell der UEFA diente der nun rechtlich verselbstständigten Deutschen Fußball-Liga (DFL) als Vorbild für die Überarbeitung ihres Vermarktungsregimes. Dabei hatten der Zusammenbruch und die Zerschlagung des Kirch-Imperiums dazu geführt, dass der Liga ihr bisheriger Free-TV-Partner verloren ging, da SAT.1 nach der Trennung von der Pay-TV-Sparte die überteuerten Free-TV-Rechte nicht mehr länger erwerben wollte. Die gravierenden Einnahmeverluste der Profivereine riefen jedoch wiederum die Politik auf den Plan, insbesondere die Ministerpräsidenten der Länder fürchteten um die Zukunft des Vereinsfußballs und einen Verlust an regionaler Identifikation (SZ 15.05.02). Schließlich erhielt die ARD, die aufgrund der bevorstehenden Gebührendebatte vor einem Wiedererwerb der Bundesligarechte zurückschreckte, von den Medienpolitikern grünes Licht für den Vertragsabschluss (SZ 25.06.03). Diese Entwicklung hatte den Vorteil, dass die DFL nur eine moderate Reduktion ihrer Fernseheinnahmen hinnehmen musste. Zugleich erleichterte es dieser Abschluss der Liga, der Forderung der EU-Kommission nach einer Vergabe mehrerer Rechtepakete in einem transparenten Verfahren nachzukommen. Dabei kann nur ein geringer Teil der Rechte von den Clubs dezentral auf nicht-exklusiver Basis vermarktet werden, von der Liga nicht-vermarktete Rechte fallen aber an die Vereine (IP/03/1106; EG-Amtsbl. vom 30.10.2003 C 261). Die DFL-Führung war allerdings nicht bereit, grundsätzlich eine (Mit-)Inhaberschaft der Proficlubs anzuerkennen, da der DFB, um sich seine Finanzierung zu sichern, bei der rechtlichen Verselbstständigung der Profiliga darauf bestanden hatte, weiterhin Inhaber der Bundesligarechte zu sein. Folgerichtig führte die Freistellungsmitteilung zu neuen Auseinandersetzungen mit den Spitzenvereinen der Liga, die eine Rechte-Inhaberschaft der Clubs reklamieren und damit den Grundlagenvertrag zwischen DFL und DFB in Frage stellten (SZ 21.11.03). Zunächst machte der neue DFL-Vertragspartner, die Rechteagentur Infront, aber geltend, dass die Freistellung den Umfang des überlassenen Rechtepaketes reduziert habe, und nahm dies zum Anlass, den verlustreichen Vertrag mit der deutschen Profiliga zu kündigen (SZ 02.01.04).

\section{Zusammenfassung und Diskussion der Ergebnisse}

Der vorliegende Beitrag hat versucht zu zeigen, in welchem Maße die gegenwärtigen Strukturen des Sportrechtemarktes auf politische Interaktionen sektoraler und politischer Akteure zurückgehen. Dabei sollte nachgewiesen werden, dass die genuin politische Konstruktion des Sportrechtemarktes nicht als evolutionärer Prozess der Selektion effizienter Interaktionsrahmen verstanden werden kann, sondern dass politische Kalküle und Prozesse die Durchsetzung ökonomischer Rationalitäten auf dem Rechte- 
markt vielfach gebrochen haben. Aus der vorliegenden Darstellung geht hervor, dass das Repertoire politischer Interventionen auf dem Sportrechtemarkt nicht nur die Definition und Zuweisung von Verfügungsrechten, sondern auch die mehr oder weniger direkte Forcierung einzelner Vertragsabschlüsse der Rechteinhaber mit den öffentlich-rechtlichen Anstalten umfasst.

An dieser Stelle soll noch einmal der Einfluss politischer Faktoren und Prozesse auf den Sportrechtemarkt verdichtet dargestellt werden. Diese zeigen sich nicht nur in den gegenwärtigen Strukturen, die durch die Überlagerung teilweise konkurrierender Politikansätze geprägt sind, sondern auch in den Marktergebnissen auf dem Sportrechtemarkt.

Aus der Rekonstruktion ging hervor, dass die Triebkräfte der politischen Konstruktion des Sportrechtemarktes auf sektoraler Seite die Konkurrenz der Medienunternehmen um attraktive Programminhalte, die durch die technische Dynamik des audiovisuellen Sektors noch einmal verschärft wurde, und das Interesse der Sportrechteinhaber an einer Maximierung ihrer Einnahmen waren, das durch sportinterne Verteilungskämpfe überlagert wurde. Es entspricht den Überlegungen Fligsteins (2001), dass die sektoralen Akteure versuchten, den Sportrechtemarkt nach der Liberalisierung der Fernsehmärkte unter Mobilisierung politischer Macht oder rechtlicher Ressourcen in ihrem Interesse zu strukturieren - und ggf. ökonomische Kalküle zu durchbrechen. Die Austragung der Konflikte in konkurrierenden Politikarenen, die unterschiedlichen Policy-Paradigmen verpflichtet und in unterschiedlichem Maße offen für die sektoralen Interessen waren, hatte dabei erheblichen Einfluss auf die Politikergebnisse. Ebenso spielten aber auch Gelegenheitsfenster bzw. die Interaktion der Auseinandersetzung mit anderen (sport-)politischen Entscheidungen eine Rolle für Prozessverläufe und -ergebnisse.

Die öffentlich-rechtlichen Anbieter nutzten erfolgreich einerseits sich bietende Gelegenheitsfenster, andererseits korrespondierende Policy-Präferenzen politischer Akteure, die an der Tradition eines starken „public service“-Sektors festhalten wollten, um ihre Beschaffungsinteressen mit den Zugangsinteressen einer breiten Zuschauerschaft zu verknüpfen. Zentral für die weit reichende Einschränkung der Verfügungsrechte der Sportrechteinhaber war dabei nicht zuletzt die Zuspitzung der öffentlichen Debatte durch die öffentlich-rechtlichen Rundfunkanstalten, die es den politischen Akteuren nahezu unmöglich machte, nicht zu gesetzlichen Interventionen zu greifen. Gegenüber der erfolgreichen Mobilisierung der Öffentlichkeit erwies sich das sportpolitische Interesse an einer Einnahmemaximierung des organisierten Sports als nicht durchsetzungsfähig.

Während die Zugangsinteressen der Sportfans auch im US-amerikanischen Kontext politische Reaktionen nach sich gezogen haben (Cox 1995), wird der unabhängige Einfluss institutioneller Parameter der Entscheidungsarenen auf die Definition des Umfangs der Verfügungsrechte der Sportrechteinhaber am hohen Niveau der deutschen und europäischen Regelungen deutlich. So privilegiert die Existenz einer spezialisierten medienpolitischen Entscheidungsarena auf nationaler und europäischer Ebene die Interessen der Nachfrager nach attraktiven Senderechten beim Zugang zu den Entscheidungsprozessen. Eine Bearbeitung des Kurzberichterstattungsproblems durch den Bund hätte mit Sicherheit zu einer stärkeren Berücksichtigung der Einnahmeinteressen des Sports geführt. Dagegen führte das gemeinsame Interesse der Bundesländer an einer Verteidigung ihres Kompetenzbereiches dazu, dass die Bundesländer sich inhaltlich bei der Ausgestaltung des Kurzberichterstattungsrechts an presserechtlichen Regelungen orientierten, um ihre Kompetenz bei der Regelung von Programmbeschaffungsfragen zu legitimieren. Der Bundesgesetzgeber fühlte sich aus sportpolitischen Erwägungen dagegen eher den Einnahmeinteressen der Sportverbände verpflichtet und schlug eine 
für die Rechteinhaber weitaus weniger einschneidende Lösung vor. Das Interesse der Bundesländer an einer Verteidigung ihres Kompetenzbereiches resultierte jedoch darin, dass die Länder an ihrer Koalition gegen den Bund festhielten und ihr Gesetzgebungsvorhaben realisierten.

Die erfolgreiche Durchsetzung einer europäischen Schutzlistenregelung geht dagegen sowohl auf die Präferenz eines beträchtlichen Teils der Europa-Parlamentarier für einen starken „public service“-Sektor als auch auf die institutionellen Eigeninteressen des Parlaments an einer sichtbaren Output-Legitimation zurück. Diese Policy-Präferenzen des EU-Parlaments konnten freilich erst aufgrund der institutionellen Reformen des Maastrichter Vertrages wirksam werden. Ohne diese veränderten institutionellen Parameter hätte die novellierte Fernsehrichtlinie wahrscheinlich keine Schutzlistenregelung enthalten.

Der Einfluss der Segmentierung von Politikarenen auf den Verlauf und das Ergebnis der Konstitution des Sportrechtemarktes wird vor allem bei den wettbewerbsrechtlichen Auseinandersetzungen deutlich. Sowohl Bundeskartellamt als auch die Europäische Wettbewerbsdirektion schlossen sich einer orthodoxen wettbewerbsrechtlichen Interpretation der Kollektivvermarktungssysteme an. Dies löste heftige Konflikte mit der Sportpolitik aus, da die wettbewerbsrechtliche Definition von Kooperations- und Austauschbeziehungen im Sportsektor das Veranstaltermonopol der Sportverbände und damit einen der Grundpfeiler des europäischen Sportmodells bedroht. Während die BKartA-Entscheidung durch den Gesetzgeber - dank eines für den DFB außerordentlich günstigen Gelegenheitsfensters - „korrigiert“ wurde, erwiesen sich die institutionellen Hürden auf europäischer Ebene als weitaus höher. Die Wettbewerbsdirektion konnte ihren Regulierungsansatz trotz der Kritik der nationalen Sportpolitik an der Infragestellung von Solidaritätsmechanismen weiter verfolgen, weil die Konstitutionalisierung des Wettbewerbsrechts als institutioneller Vetopunkt zu ihren Gunsten wirkte. Die Kommission zeigte sich erst pragmatischer, als die Auseinandersetzung um ihren Liberalisierungskurs im Sport nach dem Bosman-Urteil eskalierte. Der konzertierte sportpolitische Widerstand der Mitgliedstaaten veranlasste die EU-Kommission dazu, die Auseinandersetzungen mit den Sportverbänden zunächst zu deeskalieren, indem sie ihnen nur begrenzte Zugeständnisse abrang.

Im Hinblick auf den Einfluss politischer Faktoren auf die Strukturen des Sportrechtemarktes ist festzuhalten, dass sich auf diesem Beschaffungsmarkt als Ergebnis der Konstitution des Sportrechtemarktes in unterschiedlichen Politikarenen nun teilweise konkurrierende Politikansätze überlagern, dank der der Sportrechtemarkt den mit Abstand am stärksten regulierten Programmbeschaffungsmarkt für attraktive Programminhalte darstellt. Insbesondere dem Fußball ist damit die konkurrenzlose Attraktivität seines Produktes zum Verhängnis geworden. Das hohe Regulierungsniveau wird dabei insbesondere im Vergleich zum Filmrechtemarkt als dem zweiten zentralen Programmbeschaffungsmarkt deutlich.

\section{Fazit: Der Einfluss politischer Faktoren auf Marktstrukturen und -ergebnisse}

Der Sportrechtemarkt ist zwar unbestritten einer der kompetitivsten Beschaffungsmärkte des audiovisuellen Sektors. Die hohen Regulierungsstandards verhindern aber die ungesteuerte Durchsetzung ökonomischer Rationalitäten der Einnahmemaximierung der Sportrechteinhaber. Die Verfügungsrechte der Sportrechteinhaber wurden zugunsten der Zugangsinteressen der Allgemeinheit spürbar eingeschränkt. Dies hat das Einnahmepotenzial des Sports erheblich geschmälert ebenso wie die wettbewerbsrecht- 
lichen Regulierungen der Europäischen Kommission. Diese hat ihren Liberalisierungsansatz zwar vorerst darauf beschränkt, die wettbewerbsbeschränkenden Effekte des Sportrechtemarkts für den audiovisuellen Sektor zu minimieren. Während aus sportpolitischen Erwägungen Kollektivvermarktungssysteme trotz der ablehnenden Haltung der Wettbewerbsbehörden weiter aufrechterhalten werden, vermindert die von der Kommission durchgesetzte detaillierte Regulierung der Vermarktungspraktiken der Sportrechteinhaber über die Durchbrechung des Exklusivitätsprinzips ebenfalls den wirtschaftlichen Wert von Sportübertragungsrechten.

Für Sportpolitik und -verbände ist jedoch vor allem beunruhigend, dass die wettbewerbsrechtlichen Interventionen die zentrifugalen Kräfte innerhalb des Profifußballs gestärkt haben. Während die Einnahmepotenziale der Sportrechteinhaber verringert wurden, hat der Profifußball im Zuge der Liberalisierung der Fernsehmärkte deutlich den Charakter einer Unterhaltungsindustrie angenommen. Die Zuweisung der Verfügungsrechte an die Clubs stärkt dabei vor allem die Einnahmeinteressen der (Spitzen)Profivereine. Dies gefährdet traditionelle Solidarmechanismen im Sport und widerspricht dem sportökonomischen Konzept einer assoziativen Konkurrenz im Ligasport. Aus sportökonomischer Sicht ist das emergente Regulierungsregime also nicht als effizient anzusehen. Dabei gilt es zu vergegenwärtigen, dass eine vollständige Lösung des Profisports vom Amateursport und die Übernahme des amerikanischen Modells geschlossener Profiligen ebenfalls kaum zu realisieren sein wird, weil der Profisport damit seine notwendige sportpolitische Unterstützung verlieren würde. Die sportpolitischen Interventionen zugunsten einer wettbewerbsrechtlichen Sonderbehandlung des Sports resultieren wesentlich aus dem Bestreben, die traditionellen Sportstrukturen zu bewahren, selbst wenn diese faktisch schon beträchtlich erodiert sind.

Auf der Ebene der Marktergebnisse wird die Relevanz politischer Faktoren für den Sportrechtemarkt vor allem daran sichtbar, dass die öffentlich-rechtlichen Anstalten überhaupt Verträge über einige der attraktivsten Sportrechte abschließen konnten. Zum einen entschlossen sich die Sportverbände unter der Drohung gesetzlicher Interventionen oder aufgrund der Erwartungen ihrer sportpolitischen Adressaten, Verträge mit den öffentlich-rechtlichen Anstalten abzuschließen oder von einer exklusiven Pay-TV-Verwertung abzusehen. Zum anderen hat die Einschränkung der Verfügungsrechte der Sportrechteinhaber einen wirtschaftlichen Wertverlust zur Folge. Die Schutzlistenregelung verhindert, dass über eine Pay-TV-Verwertung die Konsumentenrenten internalisiert werden können. In Verbindung mit der gegenwärtigen Krise des Werbemarktes bringt dies die öffentlich-rechtlichen Anstalten als ernst zu nehmenden Bieter auf dem Sportrechtemarkt wieder ins Spiel. Schließlich wird die Relevanz politischer Faktoren für den Sportrechtemarkt daran sichtbar, dass die Bundesländer ihren bestehenden Einfluss auf die Beschaffungspolitik der öffentlich-rechtlichen Anstalten genutzt haben, um aufgrund standort- und sportpolitischer Erwägungen politische Preisbildungen auf dem Sportrechtemarkt herbeizuführen. Diese Eingriffe in den Marktmechanismus sind wohlfahrtsökonomisch besonders problematisch, insbesondere weil die Ergebnisse dieser Preisbildungen auf den Gebührenzahler abgewälzt werden.

Im Hinblick auf den theoretischen Hintergrund des vorliegenden Beitrags bleibt zu fragen, ob der Sportrechtemarkt durch die politischen Interaktionen tatsächlich hinreichend stabilisiert worden ist. Dies kann für die Definition des Umfangs der Verfügungsrechte bejaht werden. Die Sportverbände praktizieren aufgrund der heftigen Reaktionen der Öffentlichkeit auf den Abschluss von Pay-TV-Verträgen inzwischen weitgehend eine Vermarktungspolitik, die den Regelungszielen der Schutzlistenbestimmung entspricht. Darüber hinaus hat die Marktentwicklung - das Platzen der Digitalblase und 
die zumindest in Deutschland erfolgte Erosion vertikal integrierter Unternehmensstrukturen - dazu geführt, dass die Nachfrager nicht mehr auf den Erwerb umfassender Rechtepakete drängen. Sie bestehen zwar auf der Aufrechterhaltung klarer Verwertungshierarchien, betreiben aber nicht mehr - wie etwa die Kirch-Gruppe - die konsequente Verknappung von Nachverwertungsrechten. Die Sportrechteinhaber sind ihrerseits gezwungen, ihre Einnahmen über Zweit- und Drittverwertungen zu maximieren. Ungeachtet dieser Entspannung auf den Beschaffungsmärkten engagieren sich die öffentlich-rechtlichen Anstalten für die Anerkennung eines Kurzberichterstattungsrechts auf europäischer Ebene. Dieses Vorhaben trifft zwar auf die Unterstützung des Europaparlaments. Ein europäischer "news access“ wird aber von vornherein vermutlich nur als entgeltliches Zitierrecht angelegt sein. Auf der anderen Seite versuchen insbesondere ARD und ZDF, die Definition einer verbindlichen europäischen Schutzliste durchzusetzen, weil die europaweite Satellitenübertragung von Sportveranstaltungen ihrer Ansicht nach das Territorialprinzip bei der Rechtevergabe in Frage stellt. Diese Forderung wird jedoch weder vom Europaparlament noch von der Kommission geteilt (EUKommission 2003).

Die zentralen künftigen Auseinandersetzungen auf dem Sportrechtemarkt werden sich jedoch vermutlich um die Zuweisung von Verfügungsrechten drehen, da hier bislang kein Konsens über die Rolle der Sportverbände bei der Vermarktung und die Notwendigkeit sportspezifischer Kartellbildungen besteht. Während die Sportpolitik sich im Sinne der Bewahrung des europäischen Sportmodells für die wettbewerbsrechtliche Sonderbehandlung des Sports engagiert, steht die EU-Kommission der Bildung von Vermarktungskartellen weiterhin kritisch gegenüber und erkennt auch kein Veranstaltermonopol der Sportverbände an (Ungerer 2003). Die Profivereine verstehen sich dagegen in wachsendem Maße als "eigentliche" Veranstalter im Fußball. Angesichts der wirtschaftlichen Krise im Profifußball könnten die Verteilungskämpfe zwischen Profibereich und Sportverbänden die Frage der Veranstaltereigenschaft der Verbände noch einmal eskalieren lassen. Die wettbewerbsrechtlichen Interventionen haben es den Profivereinen bereits ermöglicht, sich gegenüber den Sportverbänden zu emanzipieren. Dem steht freilich die Stärkung der zentrifugalen Kräfte innerhalb der Ligen gegenüber, die ebenfalls zu weiteren Auseinandersetzungen über die Vermarktungsarrangements in den Ligen führen könnten.

Welche Einsichten folgen aus der vorliegenden Rekonstruktion für ein Verständnis von Kommerzialisierungsprozessen im Medienbereich? Nach Ansicht des Verfassers besteht der eigenständige Beitrag der interaktionsorientierten Perspektive auf die Konstruktion von Märkten darin, den Prozess der Kommerzialisierung und Marktkonstitution weder als evolutiven Selektionsprozess effizienter institutioneller Interaktionsrahmen noch als ungesteuerte und damit zwangsläufige „Vermarktlichung“ zu konzeptionalisieren. Auch wenn Medienmärkte zunehmend dereguliert worden sind, sind sie politisch konstruiert und bleiben eingebettet in ein umfassenderes Institutionengefüge. Dies prägt nachhaltig die Kalküle und Interaktionen der Marktteilnehmer und damit die Marktergebnisse, so dass die Rekonstruktion der Marktkonstitution für ein Verständnis aktueller Marktinteraktionen von Relevanz ist. Dabei kennt die ökonomische Soziologie natürlich auch Märkte, deren „Entbettung“ derart vorangeschritten ist, dass die Marktakteure und ihre Interaktionen soweit simplifiziert und reduziert wurden, dass die entstandenen Märkte tatsächlich neoklassische Züge tragen. Vom Standpunkt der ökonomischen Soziologie ist dies jedoch ein äußerst voraussetzungsreiches und erklärungsbedürftiges Ergebnis (MacKenzie/Millo 2003), das für Medienmärkte nur eingeschränkt zutreffen dürfte. 
Der vorliegende Beitrag hat versucht, den eigenständigen Erklärungsbeitrag einer ökonomischen Soziologie oder politischen Ökonomie von Medienmärkten an einem vergleichsweise leicht zugänglichen Untersuchungsgegenstand aufzuzeigen. Es spricht Einiges dafür, diese genuin interdisziplinäre Untersuchungsperspektive auf weitere aktuelle Prozesse der Marktkonstitution anzulegen. $\mathrm{Zu}$ denken wäre hier vor allem an die bereits wissenschaftlich gut erschlossenen Auseinandersetzungen um die Anpassung urheberrechtlicher Bestimmungen an die Bedingungen der Digitalisierung und Globalisierung, die Deregulierung und Digitalisierung der Übertragungswege oder - gleichsam als einen „evergreen“ polit-ökonomischer Untersuchungen - die gegenwärtige Gebührendebatte.

\section{Literatur}

Aminzade, R. (1992): Historical Sociology and Time. In: Sociological Methods \& Research 20, S. $456-480$.

Amsinck, M. (1997): Der Sportrechtemarkt in Deutschland: Ursachen und Konsequenzen der Gründung einer Sportrechteagentur von ARD und ZDF. In: Media Perspektiven (2), S. 62-72.

Badura, P. (1989): Das Recht auf Kurzberichterstattung. In: Zeitschrift für Urheber- und Medienrecht 33, S. 317-326.

Barros, C. P.; Ibrahimo, M.; Szymanski, S. (eds.) (2002): Transatlantic Sport: The Comparative Economics of North American and European Sports, Cheltenham; Northhampton: Elgar.

Bates, R. H.; Greif, A.; Levi, M.; Rosenthal, J.-L.; Weingast, B. R. (1998): Introduction. In: Bates, R. H.; Greif, A.; Levi, M.; Rosenthal, J.-L.; Weingast, B. R.: Analytic Narratives, Princeton, NJ: Princeton University Press, S. 3-22.

Beck, H.; Prinz, A. (1998): Sport im Pay-TV: Ein Fall für die Medienpolitik? In: Wirtschaftsdienst 78, S. 224-231.

Berg, H.; Rott, A. U. (2000): Eintritts- und Mobilitätsbarrieren im deutschen Fernsehmarkt: Das Beispiel tm3, Dortmunder Diskussionsbeiträge zur Wirtschaftspolitik Nr. 98, Dortmund: Universität Dortmund.

Blain, N.; Boyle, R.; O’Donnell, H. (1993): Sport and National Identity in the European Media, Leicester et al.: Leicester University Press.

Boardman, A. E.; Hargreaves-Heap, S. P. (1999): Network Externalities and Government Restrictions on Satellite Broadcasting of Key Sporting Events. In: Journal of Cultural Economics 23, S. $167-181$.

Brown, A. (2000): European Football and the European Commission: Governance, Participation and Social Cohesion - Towards a Policy Research Agenda. In: Soccer and Society 1, S. 129-150.

Bundesregierung (2002): 10. Sportbericht der Bundesregierung. Bundestags-Drucksache 14/9517.

Cairns, J.; Jennett, N.; Sloane, P. J. (1986): The Economics of Professional Team Sports: A Survey of Theory and Evidence. In: Journal of Economic Studies 13, S. 3-80.

Cameron, S. (1997): Regulation of the Broadcasting of Sporting Events. In: Economic Affairs 17(3), S. 37-41.

Chung, D.-N. (2001): Legitimationsgrundlagen und Zukunftschancen des öffentlich-rechtlichen Fernsehens in Deutschland: Technologische und ökonomische Aspekte und ein Leistungsvergleich im westeuropäischen Kontext, Frankfurt am Main et al.: Lang.

Conn, D. (1999): The new Commercialism. In: Hamil, S.; Michie, J.; Oughton, C. (eds.): A Game of two Halves? The Business of Football, London: Mainstream.

Cowie, C.; Williams, M. (1997): The Economics of Sports Rights. In: Telecommunications Policy 21, S. 619-634.

Cox, P. M. (1995): Flag on the Play? The Siphoning Effect on Sports Television. In: Federal Communications Law Journal 47, S. 571-591.

Dabscheck, B. (2003): International Unionism's Competitive Edge: FIFPro and the European Treaty. In: Relations Industrielles/Industrial Relations 58, S. 85-108. 
Diesbach, M. (1998): Pay-TV oder Free-TV: Zur Zulässigkeit der verschlüsselten Exklusivübertragung sportlicher Großereignisse, Baden-Baden: Nomos.

DiMaggio, P. (1994): Culture and Economy. In: Smelser, N.J.; Swedberg, R. (eds.): The Handbook of Economic Sociology, Princeton: Princeton University Press, S. 27-57.

Dobson, S.; Goddard J. (2001): The Economics of Football, Cambridge: Cambridge University Press.

Downward, P.; Dawson, A. (2000): The Economics of Professional Team Sports, London; New York: Routledge.

Egger, A.; Stix-Hackl, C. (2002): Sports and Competition Law: A Never Ending Story? In: European Competition Law Review 23, S. 81-91.

Eisenberg, C. (1997): Fussball, soccer, calcio, München: DTV.

El-Hodiri, M.; Quirk, J. (1971): An Economic Model of a Professional Sports League. In: Journal of Political Economy 70, S. 1302-1319.

Elter, V.-C. (2002): Mediale Rechte im Sport. In: Galli, A.; Gömmel, R.; Holzhäuer, W.; Straub, W. (Hrsg.): Sportmanagement: Grundlage der unternehmerischen Führung im Sport aus Betriebswirtschaftslehre, Steuern und Recht für den Sportmanager, München: Vahlen, S. 253-302.

Enderle, G. (2000): Vermarktung von Fernsehübertragungsrechten im professionellen Ligasport: Sportökonomische und wettbewerbsstrategische Aspekte, Berlin: VISTAS.

EU-Kommission (1998a): Das Europäische Sportmodell: Diskussionspapier der GD X, Brüssel.

EU-Kommission (1998b): Broadcasting of Sports Events and Competition Law: An Orientation Document from the Commission's Services. In: Competition Policy Newsletter June 1998, S. $18-28$.

EU-Kommission (2003): Mitteilung der Kommission an den Rat, das Europäische Parlament, den Europäischen Wirtschafts- und Sozialausschuss und den Ausschuss der Regionen über die Zukunft der Europäischen Regulierungspolitik im audiovisuellen Bereich, Brüssel: Europäische Kommission.

Fligstein, N. (2001): The Architecture of Markets: An Economic Sociology of Twenty-First-Century Capitalist Societies, Princeton: Princeton University Press.

Fort, R.; Quirk, J. (1995): Cross-Subsidization, Incentives, and Outcomes in Professional Team Sports Leagues. In: Journal of Economic Literature 33, S. 1265-1299.

Franck, E. (1995): Die ökonomischen Institutionen der Teamsportindustrie: Eine Organisationsbetrachtung, Wiesbaden: Gabler.

Fuhr, E. W. (1976): Das Recht des Fernsehens auf freie Berichterstattung über öffentliche Veranstaltungen. In: Burkei, F.; Polter, D.-M. (Hrsg.): Rechtsfragen im Spektrum des Öffentlichen: Mainzer Festschrift für Hubert Armbruster, Berlin: Duncker \& Humblot, S. 117-136.

Gaustad, T. (2000): The Economics of Sports Programming. In: Nordicom Review 21, S. 1001-113.

Gehring, R. (2003): Aufstieg und Fall der Kirch-Gruppe, Arbeitspapiere des Instituts für Rundfunkökonomie an der Universität zu Köln, Heft 171, Köln: Universität Köln.

Gellner, W. (1990): Ordnungspolitik im Fernsehwesen: Bundesrepublik Deutschland und Großbritannien, Frankfurt am Main et al.: Lang.

Granovetter, M. (1985): Economic Action and Social Structure: The Problem of Embeddedness. In: American Journal of Sociology 91, S. 481-510.

Gruber, B. (1995): Medienpolitik der EG, Konstanz: UVK.

Giulianotti, R. (1999): Football: A Sociology of the Global Game, Cambridge; Oxford: Polity Press.

Hannamann, I. (2001): Kartellverbot und Verhaltenskoordinationen im Sport, Berlin: Duncker \& Humblot.

Hartstein, R.; Kuch, H. (1988): Gesetzliche Regelung eines Rechts auf freie Kurzberichterstattung. In: Zeitschrift für Urheber- und Medienrecht 32, S. 503-514.

Heinemann, K. (1996): Sports Policy in Germany. In: Chalip, L.; Johnson, A.; Stachura, L. (eds.): National Sports Policy: An International Handbook, Westport: Greenwood, S. 161-187.

Hellenthal, C. (2000): Zulässigkeit einer supranationalen Fußball-Europaliga nach den Bestimmungen des europäischen Wettbewerbsrecht, Frankfurt am Main: Lang.

Hoehn, T.; Szymanski, S. (1999): The Americanization of European Football. In: Economic Policy 28, S. 205-240. 
Holznagel, B. (1996): Rundfunkrecht in Europa: Auf dem Weg zu einem Gemeinrecht europäischer Rundfunkordnung, Tübingen: Mohr.

Kruse, J. (1991): Wirtschaftliche Wirkungen einer unentgeltlichen Sport-Kurzberichterstattung im Fernsehen, Baden-Baden: Nomos.

Kruse, J. (2000): Ökonomische Probleme der deutschen Fernsehlandschaft. In: Kruse, J. (Hrsg.): Ökonomische Perspektiven des Fernsehens in Deutschland, München: Fischer, S. 7-48.

Kübler, F. (1978): Massenmedien und öffentliche Veranstaltungen: Das Verhältnis der Berichterstattungsfreiheit zu privaten Abwehr- und Ausschlussrechten, Frankfurt/Main: Hessischer Rundfunk.

Lauktien, A.-T. (1992): Der Staatsvertrag zur Fernsehkurzberichterstattung, Baden-Baden: Nomos.

Lerche, P.; Ulmer, P. (1989): Kurzberichterstattung im Fernsehen: Rechtsgutachten zur Frage der Informationsbeschaffung für die Kurzberichterstattung von Sendeunternehmen über Veranstaltungen und sonstige Ereignisse von öffentlichem Interesse, Baden-Baden: Nomos.

Lütz, S.; Czada, R. (2000): Marktkonstitution als politische Aufgabe: Problemskizze und Theorieüberblick. In: Czada, R.; Lütz, S. (Hrsg.): Die politische Konstitution von Märkten, Opladen: Westdeutscher Verlag, S. 9-36.

MacKenzie, D.; Millo, Y. (2003): Constructing a Market, Performing Theory: The Historical Sociology of a Financial Derivatives Exchange. In: American Journal of Sociology 109, S. 107-145.

Majone, G. (1996): Regulating Europe, London; New York: Routledge.

Meier, H. E. (2003): Beyond Convergence: Understandig Programming Strategies of Public Broadcasters in Competitive Environments. In: European Journal of Communication 18, S. 337-365.

Meier, H. E. (2004): Solidarität und Marktmacht. In: Sport und Gesellschaft 1, S. 125-144.

Meier, W. A.; Jarren, O. (2001): Ökonomisierung und Kommerzialisierung von Medien und Mediensystem: Einleitende Bemerkungen zu einer (notwendigen) Debatte. In: Medien \& Kommunikationswissenschaft 49 (2), S. 145-194.

Monti, M. (2003): Competition Enforcement and the Interests of Consumers - a stable Link in Times of Change, Athen, 14. Februar 2003.

Noll, R. G. (1982): Major League Sports. In: Adams, W. (ed.): The Structure of American Industry, New York; London: MacMillan, S. 348-387.

Noll, R. G. (1985): Government Regulatory Behavior: A Multidisciplinary Survey and Synthesis. Noll, R.G. (ed.): In Regulatory Policy and the Social Sciences, Berkeley: University of California Press.

North, D. C. (1990): Institutions, Institutional Change, and Economic Performance, Cambridge: Cambridge University Press.

Ott, K. (2001): Kirch in der ersten Reihe. In: Süddeutsche Zeitung vom 8. März 2001.

Parlasca, S. (1993): Kartelle im Profisport: Die wettbewerbspolitische Problematik der Mannschaftssportligen Major League Baseball, National Football League und Fußball-Bundesliga, Ludwigsburg; Berlin: Wissenschaft \& Praxis.

Parlasca, S.; Szymanski, S. (2002): When the Whole is less than the Sum of the Parts: The Negative Effects of Central Marketing of Football Television Rights on Fans, Media Concentration and Small Clubs. In: Zeitschrift für Betriebswirtschaft, Ergänzungsheft 4, S. 83-104.

Parrish, R. (2003): Sports Law and Policy in the European Union, Manchester: Manchester University Press.

Petersen, J. (2001): Fußball im Rundfunk- und Medienrecht, Beck: München.

Pollack, M. A. (1997): Delegation, Agency, and Agenda Setting in the European Community. In: International Organization 51, S. 99-134.

Quirk, J.; Fort, R. (1999): Hard Ball: The Abuse of Power in Pro Team Sports, Princeton; New Jersey: Princeton University Press.

Quitzau, J. (2003): Die Vergabe der Fernsehrechte an der Fußball-Bundesliga: Wohlfahrtsökonomische, wettbewerbspolitische und sportökonomische Aspekte der Zentralvermarktung, Frankfurt am Main et al.: Lang.

Scharpf, F. W. (1994): Optionen des Föderalismus in Deutschland und Europa, Frankfurt; New York: Campus. 
Scharpf, F. W. (1999): Regieren in Europa: Effektiv und demokratisch? Frankfurt; New York: Campus.

Schaub, A. (2002): Sports and Competition: Broadcasting Rights of Sports Events, Jornada día de la competencia, Madrid, 26 February 2002.

Schellhaaß, H. M.; Enderle, G. (1999): Wirtschaftliche Organisation von Sportligen in der Bundesrepublik Deutschland, Strauß: Sport und Buch.

Schmidt, S. K. (1998): Liberalisierung in Europa: Die Rolle der Europäischen Kommission, Frankfurt; New York: Campus.

Schneider, K.-H. (2002): Die Verankerung des Sports im Gemeinschaftsrecht. In: Sport und Recht 9, S. 137-141.

Scully, G. W. (1995): The Market Structure of Sports, Chicago: University of Chicago Press.

Seither, T. (1993): Rundfunkrechtliche Grundversorgung und Kurzberichterstattungsrecht, Stuttgart: Internationales Recht.

Solberg, H. A. (2002): The Economics of Television Sport Rights: Europe and the US - A Comparative Analysis. In: Norsk Medietidsskrift 9, S. 57-79.

Spink, P. M.; Morris, P. (2000): Battle for TV Rights in Professional Football. In: Caiger, A.; Gardiner, A. (eds.): Professional Sport in the European Union: Regulation and Re-regulation, Den Hague: Asser Press, S. 165-196.

Stockmann, K. (1996): Sportübertragungsrechte und Kartellrecht am Beispiel der Europacup-Spiele. In: Zeitschrift für Internationales Privatrecht, S. 411-418.

Stolte, D. (2001): „Strukturell bedingter Zielkonflikt“: Vorlage des ZDF-Intendanten zur Positionierung des Senders. In: epd medien vom 12. Dezember 2001, S. 30-35.

Stopper, M (1997): Ligasport und Kartellrecht, Berlin: Schmidt.

Sugden, J.; Tomlinson, A. (1998): FIFA and the Contest for World Soccer, Cambridge et al.: Polity Press.

Szymanski, S. (2000): Sport and Broadcasting, Paper presented at the IEA Seminar on Economics of Sport, October 2000.

Szymanski, S.; Kuypers, T. (1999): Winners and Losers, London et al.: Penguin.

Tettinger, P. J. (1986): Das Recht des Rundfunks auf freie Berichterstattung bei Sportveranstaltungen. In: Zeitschrift für Urheber- und Medienrecht, S. 497-507.

Thelen, K. (1999): Historical Institutionalism in Comparative Politics. In: Annual Review of Political Science 2, S. 369-404.

Tonnemacher, J. (1996): Kommunikationspolitik in Deutschland, Konstanz: UVK Medien.

UEFA (1998): UEFA Champions League: Solidarität im Europäischen Fußball, Genf: UEFA.

Ungerer, H. (2003): Commercialising Sport: Understanding the TV Rights Debate, Barcelona, 2. Oktober 2003.

Van Miert, K. (2000): Markt, Macht, Wettbewerb: Meine Erfahrungen als Kommissar in Brüssel, Stuttgart; München: DVA.

Vrooman, J. (1995): A General Theory of Professional Sports Leagues. In: Southern Economic Journal 61, S. 971-990.

Waldhauser, H. (1999): Die Fernsehrechte des Sportveranstalters, Berlin: Duncker \& Humblot.

Weatherill, S. (2003): „Fair Play please!“: Recent Development in the Application of EC Law to Sport. In: Common Market Law Review 40, S. 51-93.

Weiler, P. C.; Roberts, G. R. (1998): Sports and the Law: Text, Cases, Problems, 2nd. Ed., St. Paul, Minn.: West

Williams, J. (1994): The Local and the Global in English Soccer and the Rise of Satellite Television. In: Sociology of Sport Journal 11, S. 376-397.

Wong, G. M. (2002): Essentials of Sports Law, 3rd. ed., Westport et al.: Praeger.

Zacharias, E. (1999): Going Public einer Fußball-Kapitalgesellschaft: Rechtliche, betriebswirtschaftliche und strategische Konzepte bei der Vorbereitung der Börseneinführung eines Fußball-Bundesligavereins, Bielfeld: Schmidt. 